Supplementary Information for:

\title{
Energy Storage upon Photochromic 6- $\pi$ Photocyclization and Efficient On-demand Heat Release with Oxidation
}

\section{Stimuli}

Ryosuke Asato, ${ }^{\dagger, \ddagger}$ Colin J. Martin, ${ }^{+, \ddagger}$ Takuya Nakashima, ${ }^{\dagger}$ Jan Patrick Calupitan, ${ }^{+, \ddagger}$ Gwénaël Rapenne, ${ }^{+, \ddagger, \S}$ Tsuyoshi Kawai ${ }^{\dagger}, \neq *$

† Division of Materials Science, Graduate School of Science and Technology, Nara Institute of Science and Technology, NAIST, 8916-5 Takayama-cho, Ikoma, Nara 6300192, Japan

‡ International Collaborative Laboratory for Supraphotoactive Systems, NAIST-CEMES, CNRS UPR 8011, 29 rue Jeanne Marvig, F-31055 Toulouse Cedex 4, France $\S$ CEMES, Université de Toulouse, CNRS, 29 rue Jeanne Marvig, F-31055 Toulouse Cedex 4, France.

Corresponding author: * tkawai@ms.naist.jp 
FIGURES AND TABLES

Figure S1. UV-vis spectra of $\mathbf{1 0 - 8 0}$ in acetonitrile with new absorption bands appearing upon irradiation.

Table S1. Values in $\mathrm{kJ} \mathrm{mol}^{-1}$ of the $\Delta \mathrm{H}_{\mathrm{c}-\mathrm{o}}$ of 1-8 measured by DSC and calculated using two standard terarylene basis set functionals.

Table S2. Half-lifetime of c-form towards the non-photochemical cycloreversion reaction and the ratio of remaining species.

Figure S2. Plots of activation energy vs $\Delta \mathrm{H}_{\mathrm{c}-\mathrm{o}}$ for terarylenes 1-8 and a series of representative diarylethene molecules.

Figure S3. Plots of activation energy vs $\Delta \mathrm{H}_{\mathrm{c}-\mathrm{o}}$ for terarylenes 1-8 and a series of representative MOST molecules.

Figure S4. DSC scanning spectra of compounds 1-4 and 7.

Table S3. Summary of DSC measurements.

\section{ANALYSIS OF COMPOUNDS}

Figure S5. HR-MS data of 20 (MALDI-TOF).

Figure S6. ${ }^{1} \mathrm{H}$ NMR spectrum of $20\left(600 \mathrm{MHz}, \mathrm{CDCl}_{3}, \mathrm{TMS}, 25^{\circ} \mathrm{C}\right)$.

Figure S7. ${ }^{13} \mathrm{C}$ NMR spectrum of $20\left(151 \mathrm{MHz}, \mathrm{CD}_{2} \mathrm{Cl}_{2}, \mathrm{TMS}, 25^{\circ} \mathrm{C}\right)$.

Figure S8. HR-MS data measurement and calculation result) of 40.

Figure S9. ${ }^{1} \mathrm{H}-\mathrm{NMR}$ spectrum of $40\left(600 \mathrm{MHz}, \mathrm{CDCl}_{3}, \mathrm{TMS}, 25^{\circ} \mathrm{C}\right)$.

Figure S10. ${ }^{13} \mathrm{C}$ NMR spectrum of $40\left(151 \mathrm{MHz}, \mathrm{CDCl}_{3}, \mathrm{TMS}, 25^{\circ} \mathrm{C}\right)$.

Figure S11. X-ray Structure of 40.

Table S4. Crystallographic parameters and refinement details for $\mathbf{4 0}$. 


\section{Experimental details}

${ }^{1} \mathrm{H}$ and ${ }^{13} \mathrm{C}$ NMR $(300,400$ and $600 \mathrm{MHz})$ spectra were recorded on JEOL JNM-AL300, JEOL JNMECP400 and JEOL JNMECA600 spectrometers, respectively. Recycling preparative GPC were performed on Japan Analytical Industry LC-9110NEXT. Mass spectrometry and high-resolution mass spectrometry were performed on matrix assisted laser desorption/ionization (MALDI) time of Flight (TOF) - MS spectra (Bruker Autoflex II and JEOL spiralTOF JMS-S3000). UV/Vis spectra, quantum yields of photochromic reactions $\left(\varphi_{c-o}\right.$ and $\left.\varphi_{o-c}\right)$ and photo-induced fading reaction were measured using a JASCO V-660, V-760 spectrophotometer and a Shimadzu QYM-01 set-up, respectively. Calculations were performed with the Gaussian09 package. ${ }^{1}$ We worked at the B3LYP 6-31G(d) level of theory in vacuo. For kinetic thermal analyses, the temperature was controlled by a JASCO ETC 505 T temperature controller. Heat quantity was measured using Differential scanning calorimeter, SHIMAZU DSC-60Plus and SHIMAZU TA-60WS thermal analyzer. For the demonstration of heat release system, the temperature was monitored by CHINO sheathed thermocouple (diameter $0.3 \mathrm{~mm}$ ) and Fine FHP301Npro. Thermographic images were recorded on Optris Compact spot finder IR camera Xi 400.

Compounds $1,{ }^{2} 3,{ }^{3} 5,{ }^{4} 6,{ }^{5} 7^{3}$ and $8^{6}$ were synthesized as previously reported. compounds 2, 4 and S6 were prepared according to the routes depicted in Schemes S1 and S2. Compounds $\mathbf{S 1},{ }^{7} \mathbf{S} 3,{ }^{8} \mathbf{S 4},{ }^{9} \mathbf{S} 5^{3}$ were prepared as previously reported, and compound $\mathbf{S 2}$ was commercially available. The structures have all been successfully characterized using NMR and HR-MS. NMR and MS spectra of the new final targets are in Figures S5-S10. 


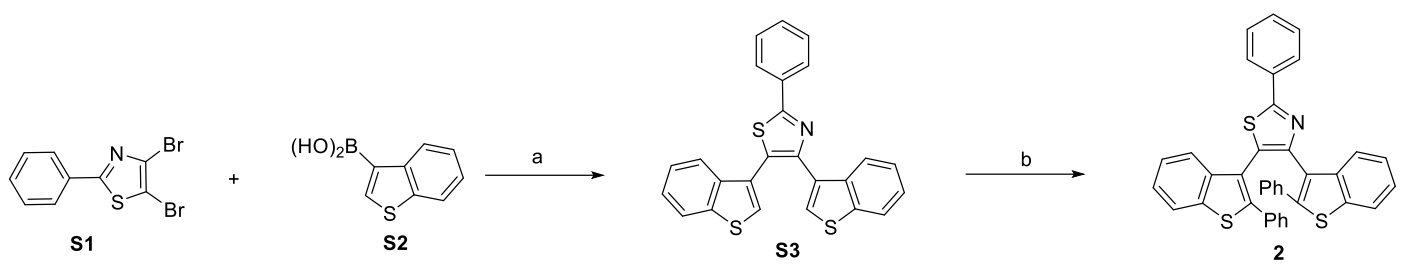

Scheme S1. Synthesis of 2: a) $\left[\mathrm{Pd}\left(\mathrm{PPh}_{3}\right)_{4}\right], \mathrm{PPh}_{3}, 2 \mathrm{M} \mathrm{K} \mathrm{K}_{3} \mathrm{OO}_{4}$, water/1,4-dioxane, $\mathrm{THF}, 24 \mathrm{~h}, 110^{\circ} \mathrm{C}$; b) Boronic acid, (' $\left.{ }^{\mathrm{Bu}}\right)_{2} \mathrm{PMeHBF}_{4}$, pivalic acid, $\mathrm{Cs}_{2} \mathrm{CO}_{3}, \mathrm{Pd}(\mathrm{OAc})_{2} 16 \mathrm{~h}, 150^{\circ} \mathrm{C}$.

4,5-bis(benzothiophen-3-yl)-2-phenylthiazole (S3): A two-necked flask was charged with 4,5-dibromo-2-phenylthiazole (S1) $(0.48 \mathrm{~g}, 2.0 \mathrm{mmol})$, benzothiophen-3-ylboronic acid (S2) (1.28 g, $4.4 \mathrm{mmol})$, triphenylphosphine (0.26 g, $1.0 \mathrm{mmol})$ and $2 \mathrm{M}$ aqueous tripotassium phosphate $(15 \mathrm{~mL})$ in 1,4-dioxane $(15 \mathrm{~mL})$. After 15 minutes of stirring under a nitrogen atmosphere, tetrakis(triphenylphosphine)palladium(0) $(0.34 \mathrm{~g}, 0.2$ mmol) was added and stirred under nitrogen atmosphere at $110^{\circ} \mathrm{C}$ for 24 hours. The organic layer was then extracted with chloroform, and the combined extracts were washed with water and dried over anhydrous sodium sulfate. The sample was then filtered, and the filtrate concentrated in vacuo. Gel Permeation Chromatography (chloroform) afforded 4,5-bis(benzothiophen-3-yl)-2-phenylthiazole (S3) as a white powder (0.59 g, $1.3 \mathrm{mmol}, 65 \%$ yield). ${ }^{1} \mathrm{H}-\mathrm{NMR}\left(300 \mathrm{MHz}, \mathrm{CDCl}_{3}, \mathrm{TMS}, 25^{\circ} \mathrm{C}\right) \delta(\mathrm{ppm})$ 7.42-7.50 (m, 7H), $7.81(\mathrm{~s}, 2 \mathrm{H}), 7.86-8.18(\mathrm{~m} 6 \mathrm{H})$. consistent with previous reports ${ }^{8}$. HRMS (MALDI-TOF): $\mathrm{m} / \mathrm{z}$ calcd. for $\mathrm{C}_{37} \mathrm{H}_{23} \mathrm{NS}_{3}[\mathrm{M}]^{+}$: 657.168; found 657.149.

4,5-Bis(2,5-diphenylthiazol-4-yl)-2-phenylthiazole (2): A two-necked flask was charged with 4,5-bis(benzothiophen-3-yl)-2-phenylthiazole (S3) $(0.10 \mathrm{~g}, 0.23 \mathrm{mmol})$, bromobenzene $(0.08 \mathrm{~g}, 0.51 \mathrm{mmol})$, di-tert-butylmethylphosphine tetrafluoroborate $(0.011 \mathrm{~g}, 0.046 \mathrm{mmol})$, cesium carbonate $(0.30 \mathrm{~g}, 0.92 \mathrm{mmol})$, palladium acetate $(0.10 \mathrm{~g}$, 
$0.046 \mathrm{mmol})$ and pivalic acid $(0.011 \mathrm{~g}, 0.11 \mathrm{mmol})$ in mesitylene $(2 \mathrm{~mL})$. The mixture was heated under reflux at $150^{\circ} \mathrm{C}$ for 16 hours. Thereafter, it was filtered through celite, extracted with ethyl acetate, and the combined extracts were washed with water and dried over anhydrous sodium sulfate. Gel Permeation Chromatography (chloroform) afforded 2 (0.019 g, $0.032 \mathrm{mmol}, 14 \%$ yield) as a colorless powder. ${ }^{1} \mathrm{H}-\mathrm{NMR}(600 \mathrm{MHz}$, $\left.\mathrm{CDCl}_{3}, \mathrm{TMS}^{\circ} 2^{\circ} \mathrm{C}\right) \delta(\mathrm{ppm}) 8.16(\mathrm{~d}, \mathrm{~J}=6.2 \mathrm{~Hz}, 2 \mathrm{H}), 7.60(\mathrm{dd}, \mathrm{J}=18.9,7.9 \mathrm{~Hz}, 2 \mathrm{H}), 7.51(\mathrm{dt}$, $J=18.6,7.2 \mathrm{~Hz}, 3 \mathrm{H}), 7.33(\mathrm{~s}$, broad, $1 \mathrm{H}), 7.25(\mathrm{~s}, 1 \mathrm{H}), 7.21-7.15(\mathrm{~m}, 2 \mathrm{H}), 7.09(\mathrm{~m}, 2 \mathrm{H})$, $6.88(\mathrm{~d}, \mathrm{~J}=8.2 \mathrm{~Hz}, 2 \mathrm{H}), 6.84(\mathrm{~d}, \mathrm{~J}=8.2 \mathrm{~Hz}, 2 \mathrm{H}), 6.69$ (s broad, $4 \mathrm{H}), 6.53$ (s broad, $2 \mathrm{H}) ;{ }^{13} \mathrm{C}$ NMR (151 MHz, $\left.\mathrm{CD}_{2} \mathrm{Cl}_{2}, \mathrm{TMS}, 25^{\circ} \mathrm{C}\right) \delta(\mathrm{ppm}) 167.0,149.0,143.1,139.8,139.5,139.1$, $138.9,134.1,133.8,133.3,130.6,129.4,129.0,128.2,128.2,128.0$ - 127.6 (5 broad overlapping peaks observed), 126.7, 126.0, 124.7, 124.5, 124.3, 124.2, 124.0, 123.4, 122.2, 121.8, 121.5. HR-MS (ESI): $\mathrm{m} / \mathrm{z}$ calcd. for $\mathrm{C}_{37} \mathrm{H}_{23} \mathrm{NS}_{3}: 577.0993\left[\mathrm{M}^{+}\right]$; found: 577.0998 .

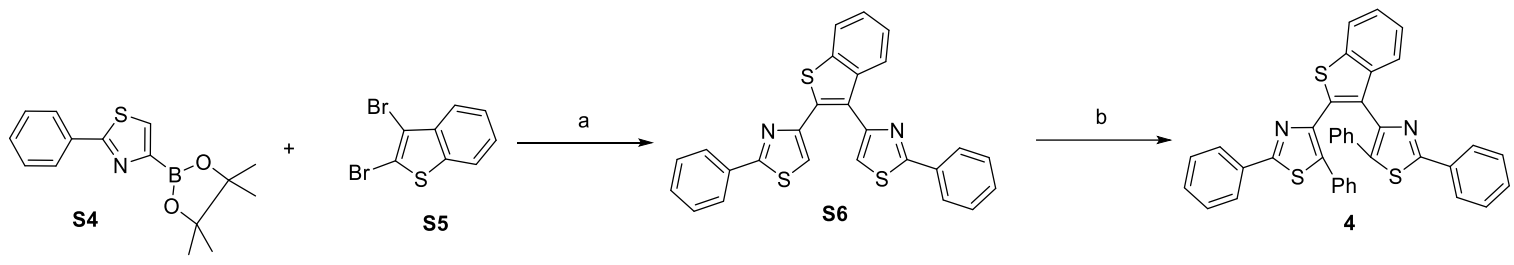

Scheme S2. Synthesis of 4: a) $\left[\mathrm{Pd}\left(\mathrm{PPh}_{3}\right)_{4}\right], P P h_{3}, 2 \mathrm{M} \mathrm{K}_{3} \mathrm{PO}_{4}$, water/1,4-dioxane, $\mathrm{THF} 24 \mathrm{~h}, 110^{\circ} \mathrm{C}$; b) Phenylboronic acid, ( $\left.{ }^{\mathrm{B}} \mathrm{Bu}\right)_{2} \mathrm{PMeHBF}_{4}$, pivalic acid, $\mathrm{Cs}_{2} \mathrm{CO}_{3}, \mathrm{Pd}(\mathrm{OAc})_{2} 16 \mathrm{~h}, 150^{\circ} \mathrm{C}$.

4,4'-(benzo[b]thiophene-2,3-diyl)bis(2-phenylthiazole) (S6): A two-necked flask was charged with 2-phenyl-4-(4,4,5,5-tetramethyl-1,3,2-dioxaborolan-2-yl)thiazole (S4) (660 $\mathrm{mg}, \quad 2.3 \mathrm{mmol}$ ), 2,3-dibromobenzo[b]thiophene (S5) (321 $\mathrm{mg} 1.1 \mathrm{mmol}$, ), triphenylphosphine $(43 \mathrm{mg}, 0.165 \mathrm{mmol}$ ) and $2 \mathrm{M}$ aqueous tripotassium phosphate (10 $\mathrm{mL}$ ) in 1,4-dioxane (10 mL). After 60 minutes of stirring under nitrogen atmosphere, 
tetrakis(triphenylphosphine)palladium(0) $(80 \mathrm{mg}, 0.047 \mathrm{mmol})$ was added and stirred under nitrogen atmosphere at $110^{\circ} \mathrm{C}$ for 24 hours. The organic layer was then extracted with chloroform and the combined extracts were washed with water and dried over anhydrous sodium sulfate. The sample was then filtered, and the filtrate concentrated in vacuo. Gel Permeation Chromatography (chloroform) afforded 4,4'-(benzo[b]thiophene2,3-diyl)bis(2-phenylthiazole) (S6) $\left(0.35 \mathrm{~g}, 0.7 \mathrm{mmol}, 70 \%\right.$ yield) as a white powder. ${ }^{1} \mathrm{H}$ NMR (300 MHz, $\left.\mathrm{CDCl}_{3}, \mathrm{TMS}, 25^{\circ} \mathrm{C}\right) \delta(\mathrm{ppm})$ 8.10-8.07 (m, 2H), 8.02-7.99 (m, 2H), 7.91$7.88(m, 1 H), 7.72-7.70(m, 1 H), 7.49-7.43(m, 7 H), 7.41-7.37(m, 2 H), 7.16(s, 1 H)$.

4,4'-(benzo[b]thiophene-2,3-diyl)bis(2,5-diphenylthiazole) (4): A two-necked flask was charged with 4,4'-(benzo[b]thiophene-2,3-diyl)bis(2-phenylthiazole) (S6) (0.20 g, 0.44 mmol), bromobenzene $(0.16 \mathrm{~g}, 1.04 \mathrm{mmol})$, di-tert-butylmethylphosphine tetrafluoroborate $(0.021 \mathrm{~g}, 0.09 \mathrm{mmol})$, cesium carbonate $(0.054 \mathrm{~g}, 1.7 \mathrm{mmol})$, palladium acetate $(0.019 \mathrm{mg}, 85 \mu \mathrm{mol})$ and pivalic acid $(0.026 \mathrm{~g}, 0.25 \mathrm{mmol})$ in mesitylene $(1.5 \mathrm{~mL})$. The mixture was heated under reflux at $150^{\circ} \mathrm{C}$ for 16 hours. Thereafter, it was filtered through celite, extracted with ethyl acetate and the combined extracts were washed with water and dried over anhydrous sodium sulfate. GPC (chloroform) afforded 4 (0.050 g, $0.083 \mu \mathrm{mol}, 16 \%$ yield) as a white powder. ${ }^{1} \mathrm{H}$ NMR $\left(600 \mathrm{MHz}, \mathrm{CDCl}_{3}, \mathrm{TMS}, 25^{\circ} \mathrm{C}\right) \delta$ (ppm) $7.86(d, J=7.9 \mathrm{~Hz}, 1 \mathrm{H}), 7.80(\mathrm{~d}, \mathrm{~J}=7.9 \mathrm{~Hz}, 1 \mathrm{H}), 7,79(\mathrm{~m}, 2 \mathrm{H}), 7.71$ (dd, J = 7.9, 1.7 $\mathrm{Hz}, 2 \mathrm{H}), 7.41-7.33(\mathrm{~m}, 8 \mathrm{H}), 7.07(\mathrm{dd}, \mathrm{J}=7.2 \mathrm{~Hz}, 1.4 \mathrm{~Hz}, 2 \mathrm{H}), 7.03(\mathrm{t}, \mathrm{J}=7.2 \mathrm{~Hz}, 2 \mathrm{H}), 7,00$ $(\mathrm{d}, \mathrm{J}=7.8 \mathrm{~Hz}, 2 \mathrm{H}), 6.96-6.91(\mathrm{~m}, 4 \mathrm{H}) ;{ }^{13} \mathrm{C} \mathrm{NMR}\left(151 \mathrm{MHz}, \mathrm{CDCl}_{3}, \mathrm{TMS}, 25^{\circ} \mathrm{C}\right) \delta(\mathrm{ppm}) 165.0$, $145.3,143.8,139.8,136.2,136.0,135.8,133.8,133.4,131.2,130.5,129.8,129.6,128.8$ 128.7, 128.6 (2 overlapping peaks), 128.4, 128.4, 128.3, 128.1, 127.7, 126.5, 126.4, 124.9, 124.5, 122.0; HR-MS (ESI): m/z calcd. for $\mathrm{C}_{38} \mathrm{H}_{24} \mathrm{~N}_{2} \mathrm{~S}_{3}: 604.1096$ [M+]; found: 604.1097. 

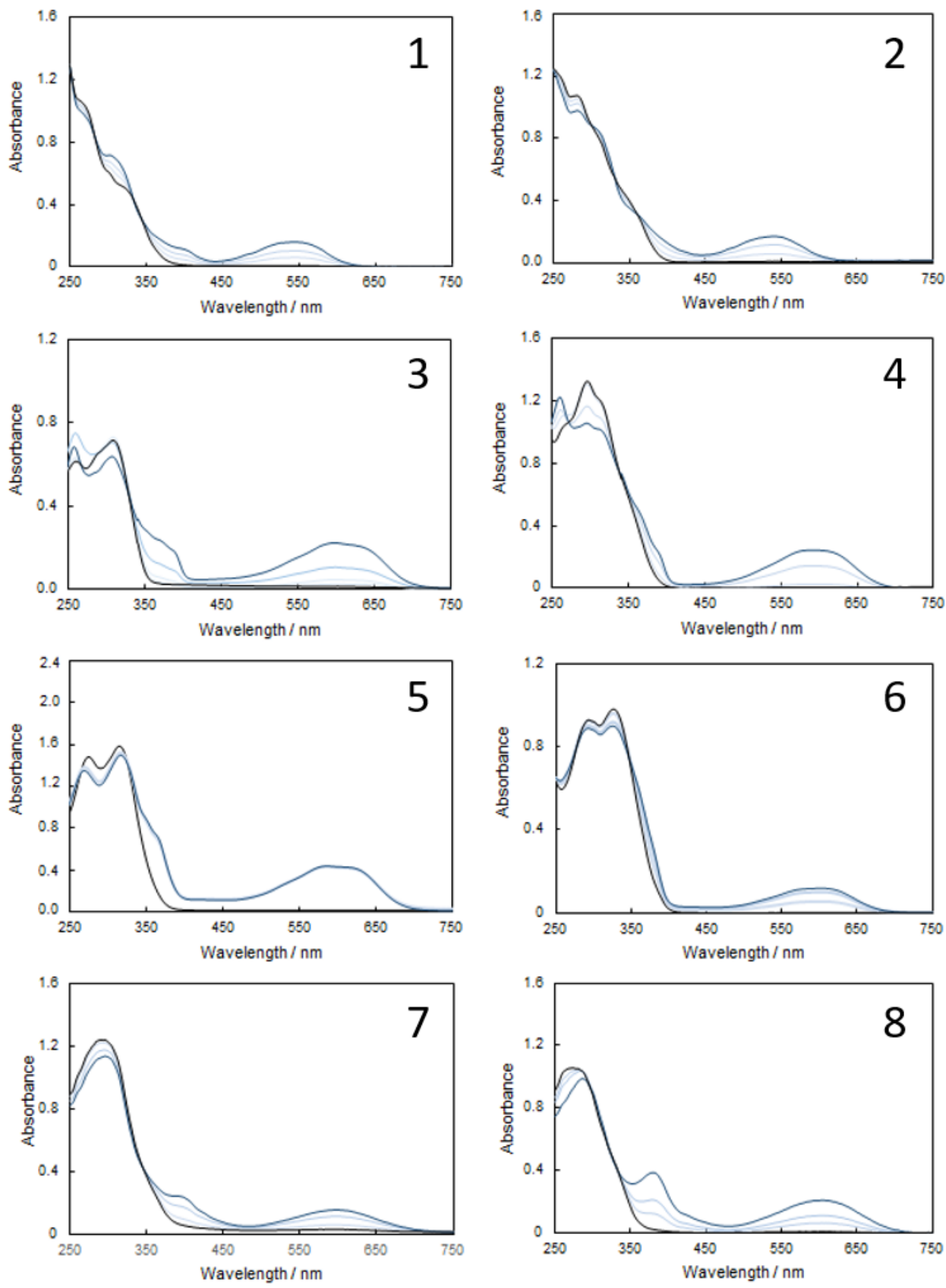

Figure S1. UV-vis spectra (black) of 10-80 in acetonitrile with new absorption bands (blue) appearing upon irradiation (UV light at $365 \mathrm{~nm}$ ). Visible light irradiation turns them back to the o-form. Concentration = (1) $4.6 \times 10^{-5} \mathrm{M}$; (2) $5.6 \times 10^{-5} \mathrm{M}$; (3) $2.5 \times 10^{-5} \mathrm{M}$; (4) $4.5 \times 10^{-5} \mathrm{M}$; (5) $4.1 \times 10^{-5} \mathrm{M}$; (6) $2.9 \times 10^{-5} \mathrm{M}$; (7) $3.0 \times 10^{-5} \mathrm{M}$; (8) $4.0 \times 10^{-5} \mathrm{M}$. 
Table S1. Values in $\mathrm{kJ} \mathrm{mol}^{-1}$ of the $\Delta \mathrm{H}_{\mathrm{c}-\mathrm{o}}$ of 1-8 measured by DSC, with the calculated values in obtained from two standard terarylene basis set functionals B3LYP/6-31G(d) and WB97X-D 6-31G(d,p).

\begin{tabular}{|c|c|c|c|}
\hline & $\Delta H_{\mathrm{c}-\mathrm{o}}, \mathrm{DSC}^{[\mathrm{a}]}$ & $\begin{array}{c}\Delta \mathrm{H}_{\mathrm{c}-\mathrm{o}}, \mathrm{DFT}^{[\mathrm{b}]} \\
\text { B3LYP/6-31G(d) }\end{array}$ & $\begin{array}{c}\left.\Delta H_{c-0}, D T^{[b]}\right) \\
\text { WB97X-D/6-31G(d,p) }\end{array}$ \\
\hline 1 & 32.7 & 76.7 & 68.7 \\
\hline 2 & 114 & 136.3 & 151.4 \\
\hline 3 & 24.8 & 74.3 & 67.9 \\
\hline 4 & 109.6 & 128.7 & 130.1 \\
\hline 5 & - & 86.9 & 83.8 \\
\hline 6 & - & 142.8 & 148.9 \\
\hline 7 & 70.6 & 98.3 & 97.5 \\
\hline 8 & - & 162.8 & 168.2 \\
\hline
\end{tabular}

[a] based on the integrated heat release in DSC profile and the conversion rate of c-form determined with UV-vis spectra. [b] is calculated from the difference in the computationally calculated 'Sum of Electronic and Thermal Free Energies of the open and closed forms of each pair of photochromic isomers. 
Table S2. Half-lifetime of c-form towards the non-photochemical cycloreversion reaction and the ratio of remaining species $(T)$

\begin{tabular}{llll}
\hline & $\mathrm{k} / \mathrm{s}^{-1}[\mathrm{a}]$ & $\mathrm{Ea}_{\mathrm{c}-\mathrm{o}} / \mathrm{kJ} \mathrm{mol}^{-1}[\mathrm{~b}]$ & $A / \mathrm{s}^{-1}[\mathrm{~b}]$ \\
\hline $\mathbf{1}$ & $1.0 \times 10^{-8}$ & 127 & $1.4 \times 10^{8}$ \\
$\mathbf{2}$ & $5.1 \times 10^{-8}$ & 106 & $4.8 \times 10^{11}$ \\
$\mathbf{3}^{[\mathrm{c}]}$ & $2.9 \times 10^{-10}$ & 137 & $5.3 \times 10^{15}$ \\
$\mathbf{4}$ & $3.8 \times 10^{-7}$ & 114 & $2.5 \times 10^{13}$ \\
$\mathbf{5}^{[\mathrm{d}]}$ & $5.8 \times 10^{-4}$ & 112 & $5.2 \times 10^{16}$ \\
$\mathbf{6}^{[\mathrm{d}]}$ & $4.9 \times 10^{-3}$ & 104 & $1.7 \times 10^{26}$ \\
$\mathbf{7}$ & $6.5 \times 10^{-7}$ & 92 & $1.6 \times 10^{10}$ \\
$\mathbf{8}$ & $1.2 \times 10^{-2}$ & 37 & $4.3 \times 10^{4}$ \\
\hline
\end{tabular}

All measurements reported in toluene. [a] rate constant of a first-order decay at $20^{\circ} \mathrm{C}$; [b] the parameters of thermal cycloreversion reaction by means of Arrhenius relationship of temperature-dependent cycloreversion kinetics following Arrhenius's equation: $\ln k=\ln A-$ $\frac{E a}{R T} ;[c]$ data from Ref.3; [d] data from Ref.5. 


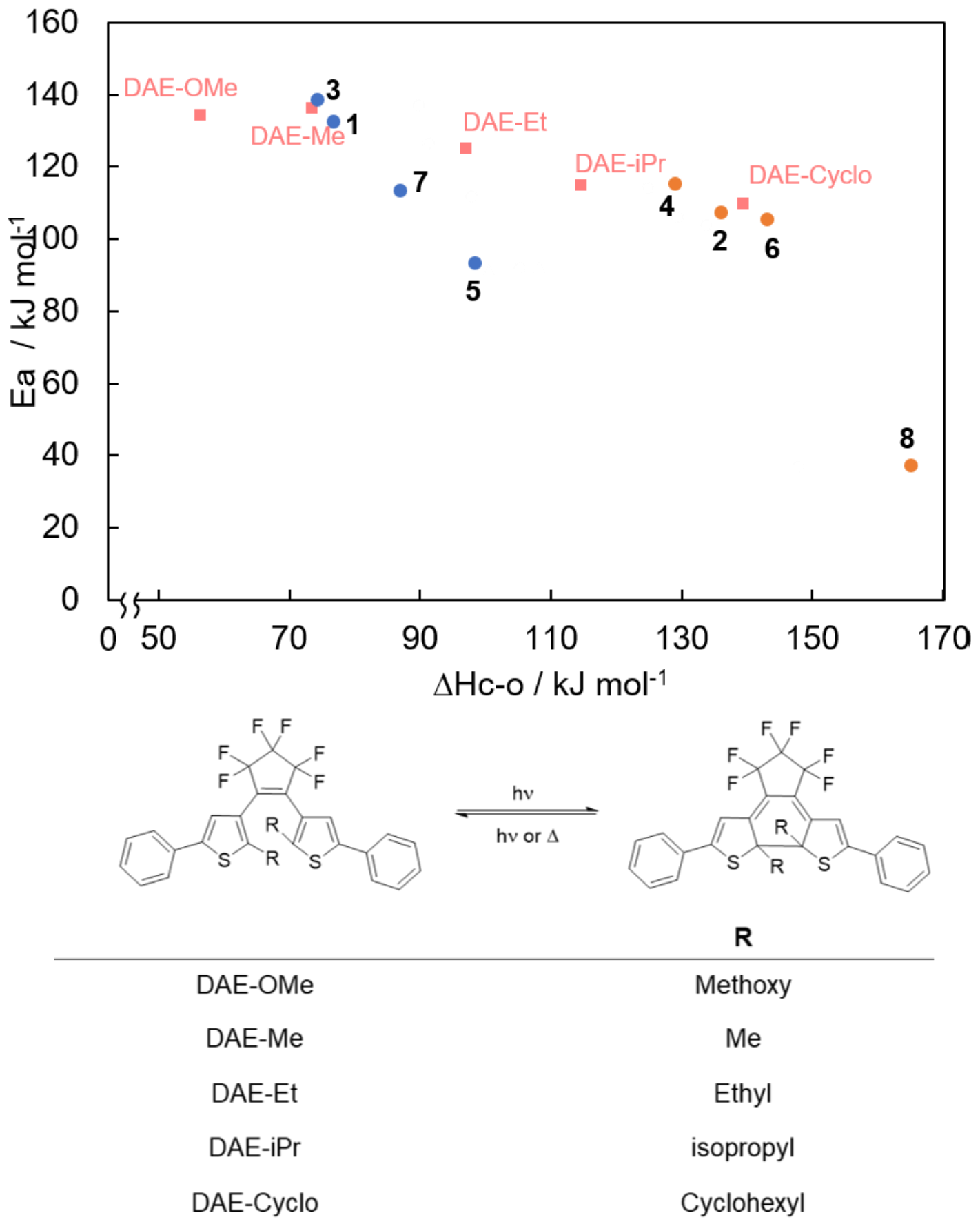

Figure S2. Plots of activation energy vs $\Delta H_{c-o}$ for terarylenes 1-8 and a series of representative diarylethene molecules shown below. Their corresponding $\Delta H_{\text {iso }}$ have been calculated by the same method using the B3LYP functional with the 6-31G(d) basis set. 

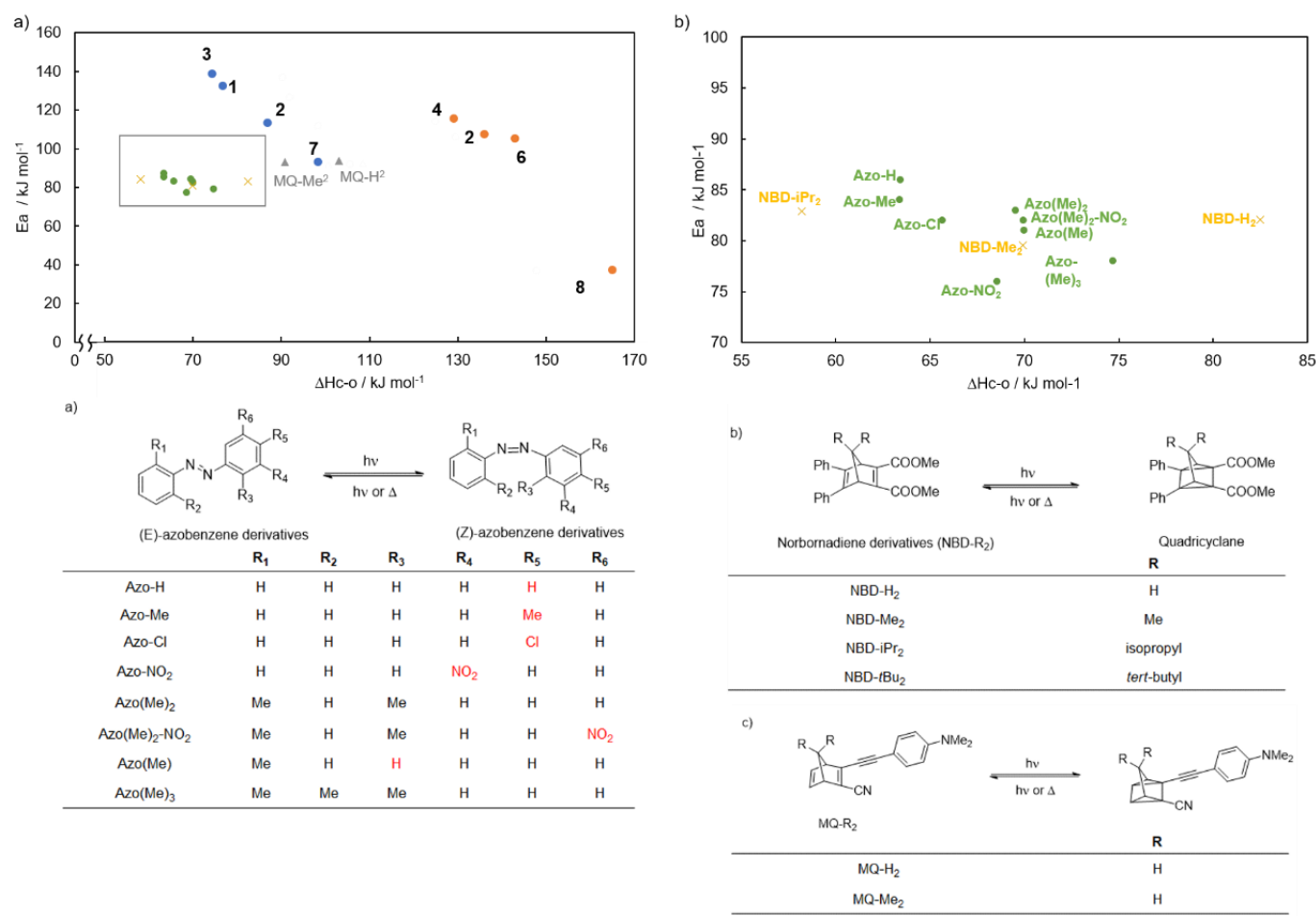

Figure S3. Plots of activation energy vs $\Delta H_{c-o}$ for terarylenes 1-8 and a series of representative MOST molecules of three types: a) azobenzenes b) norbornadienes and c) acetylenenorbornadienes. ${ }^{10,11}$ 


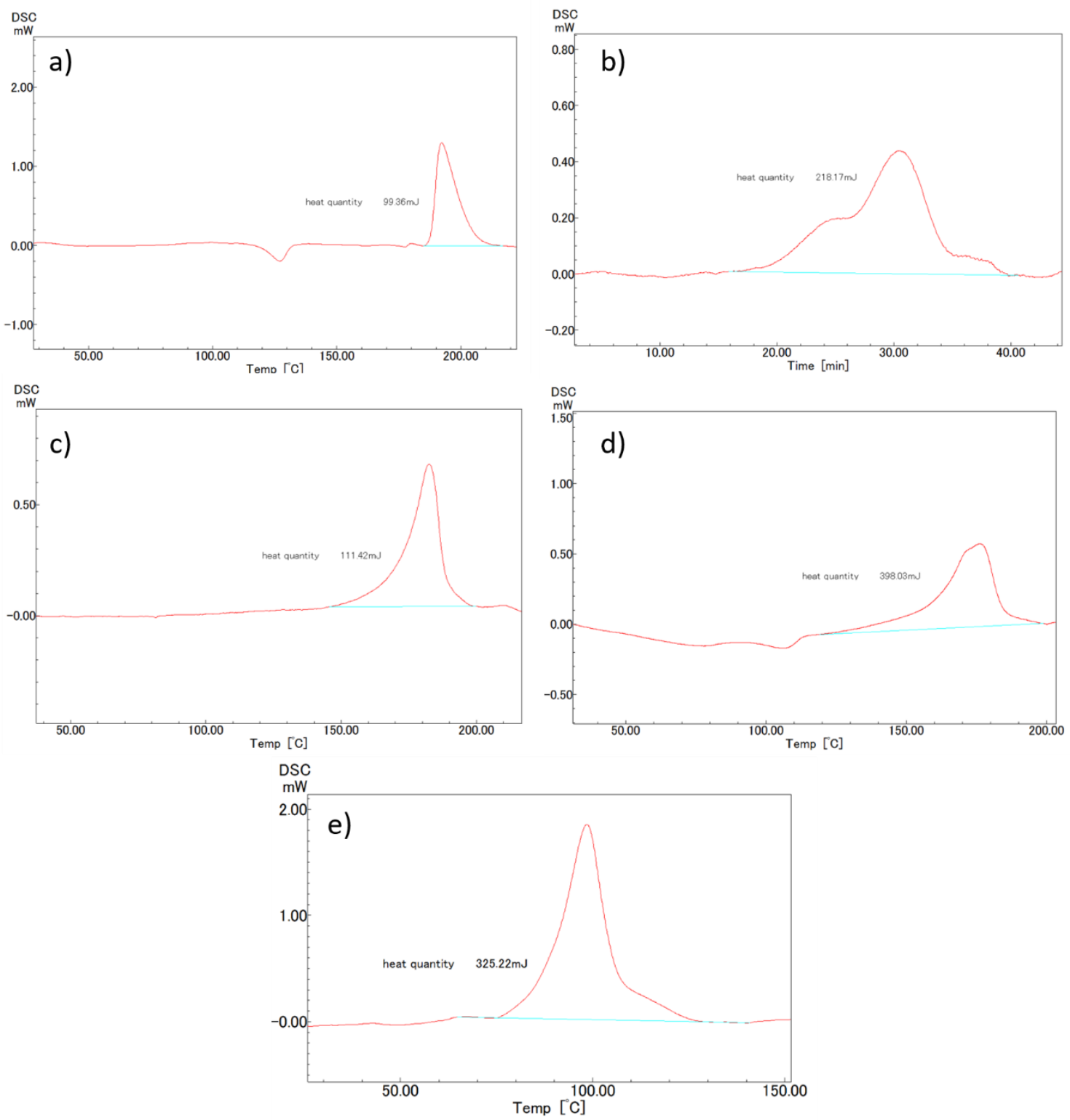

Figure S4. DSC scanning spectra of compounds 1-4 and 7. The heat quantity of the powder containing a photostationary state mixture for compounds $\mathbf{1}(a), \mathbf{2}(b), \mathbf{3}(c), \mathbf{4}(d)$ and $\mathbf{7}(e)$ were measured. The scan speed and the packed weight of powder in the DSC holder are a) $5^{\circ} \mathrm{C} \mathrm{min}^{-1}$ and $4.0 \mathrm{mg}, \mathrm{b}) 5^{\circ} \mathrm{C} \mathrm{min}^{-1}$ and $\left.2.4 \mathrm{mg}, \mathrm{c}\right) 8^{\circ} \mathrm{C} \mathrm{min}^{-1}$ and $2.5 \mathrm{mg}^{\text {d) }} 5^{\circ} \mathrm{C} \mathrm{min}^{-1}$ and $3.1 \mathrm{mg}$, e) $5^{\circ} \mathrm{C} \mathrm{min}^{-}$ 1 and $3.2 \mathrm{mg}$. 
Table S3. Summary of DSC measurements

\begin{tabular}{llll}
\hline & $\begin{array}{l}\text { Heat quantity } \\
\left(\mathrm{mJ} \mathrm{g}^{-1}\right)\end{array}$ & $\begin{array}{l}\text { Conversion rate } \\
\text { of c-form }\end{array}$ & $\begin{array}{l}\Delta \mathrm{H}_{\mathrm{c}-\text {, measured }}{ }^{[\mathrm{b}]} \\
\left(\mathrm{kJ} \mathrm{mol}^{-1}\right)\end{array}$ \\
\hline $\mathbf{1}$ & 41.4 & 0.58 & 32.7 \\
$\mathbf{2}$ & 87.3 & 0.45 & 114 \\
$\mathbf{3}$ & 35.9 & 0.70 & 24.8 \\
$\mathbf{4}$ & 124 & 0.69 & 109.6 \\
$\mathbf{7}$ & 81.3 & 0.62 & 70.6 \\
\hline
\end{tabular}

[a] data are estimated by the integration for the peak of heat release in DSC measurement with the mass of each compound. [b] data are estimated using UV-vis spectrum by comparing the $\mathbf{0} / \mathbf{c}$ form of mixture powder dissolved in acetonitrile and the o-form induced by irradiation of visible light. [c] data are estimated with conversion rate of c-form. 


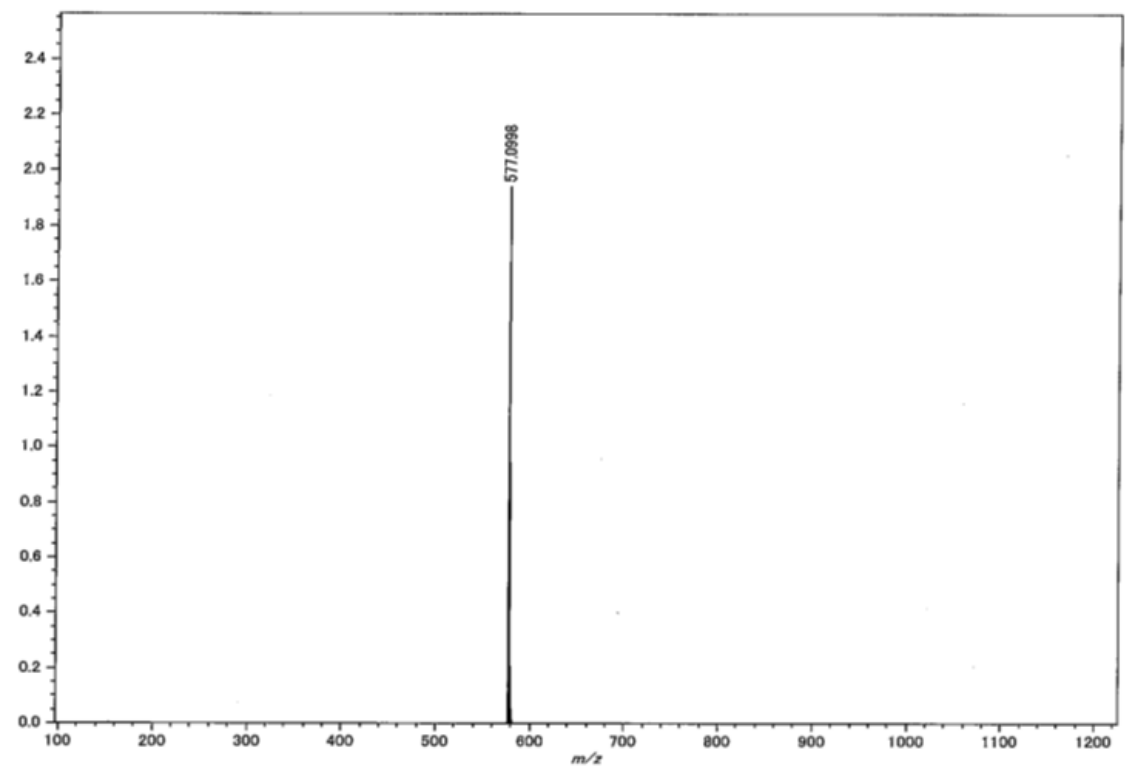

Figure S5. HR-MS data of 20 (MALDI-TOF).

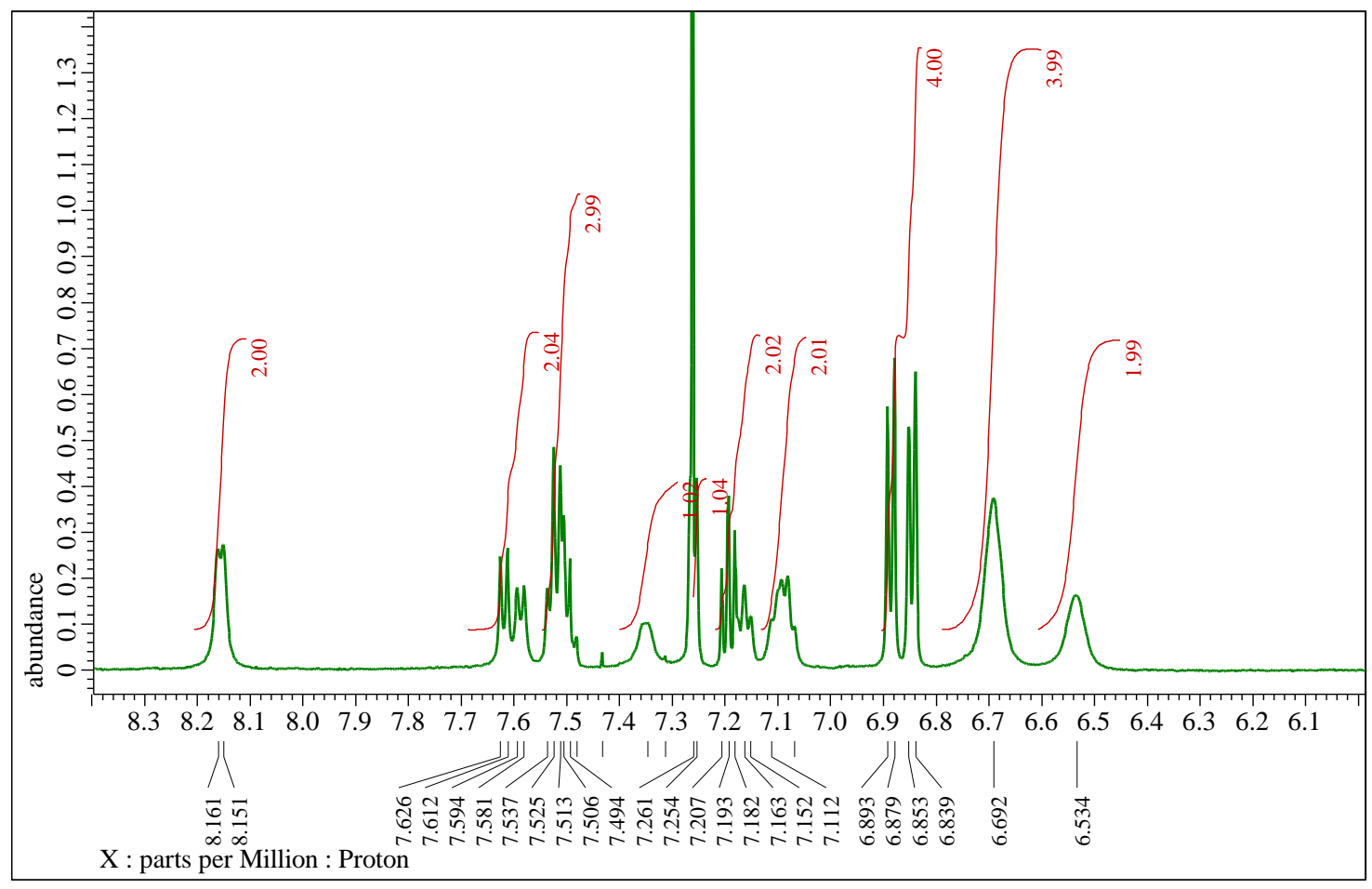

Figure S6. ${ }^{1} \mathrm{H} N \mathrm{NR}$ spectrum of $20\left(600 \mathrm{MHz}, \mathrm{CDCl}_{3}, \mathrm{TMS}, 25^{\circ} \mathrm{C}\right)$. 


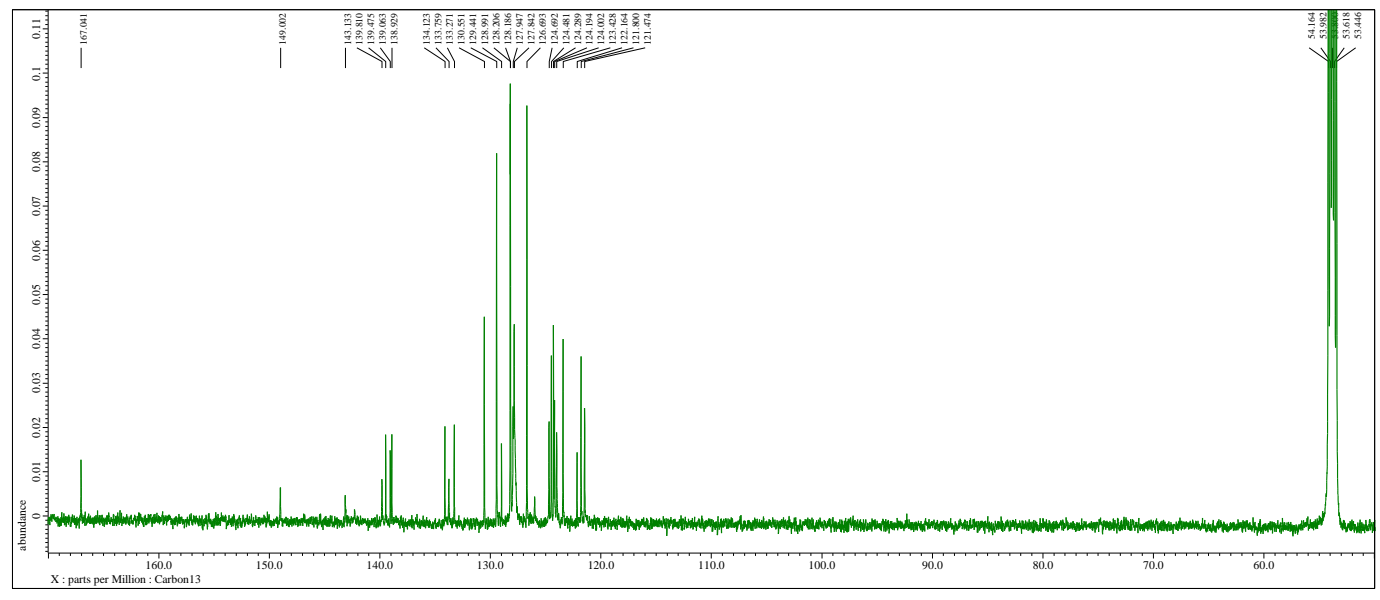

Figure S7. ${ }^{13} \mathrm{C} N M R$ spectrum of $20\left(151 \mathrm{MHz}, \mathrm{CD}_{2} \mathrm{Cl}_{2}, \mathrm{TMS}, 25^{\circ} \mathrm{C}\right)$.

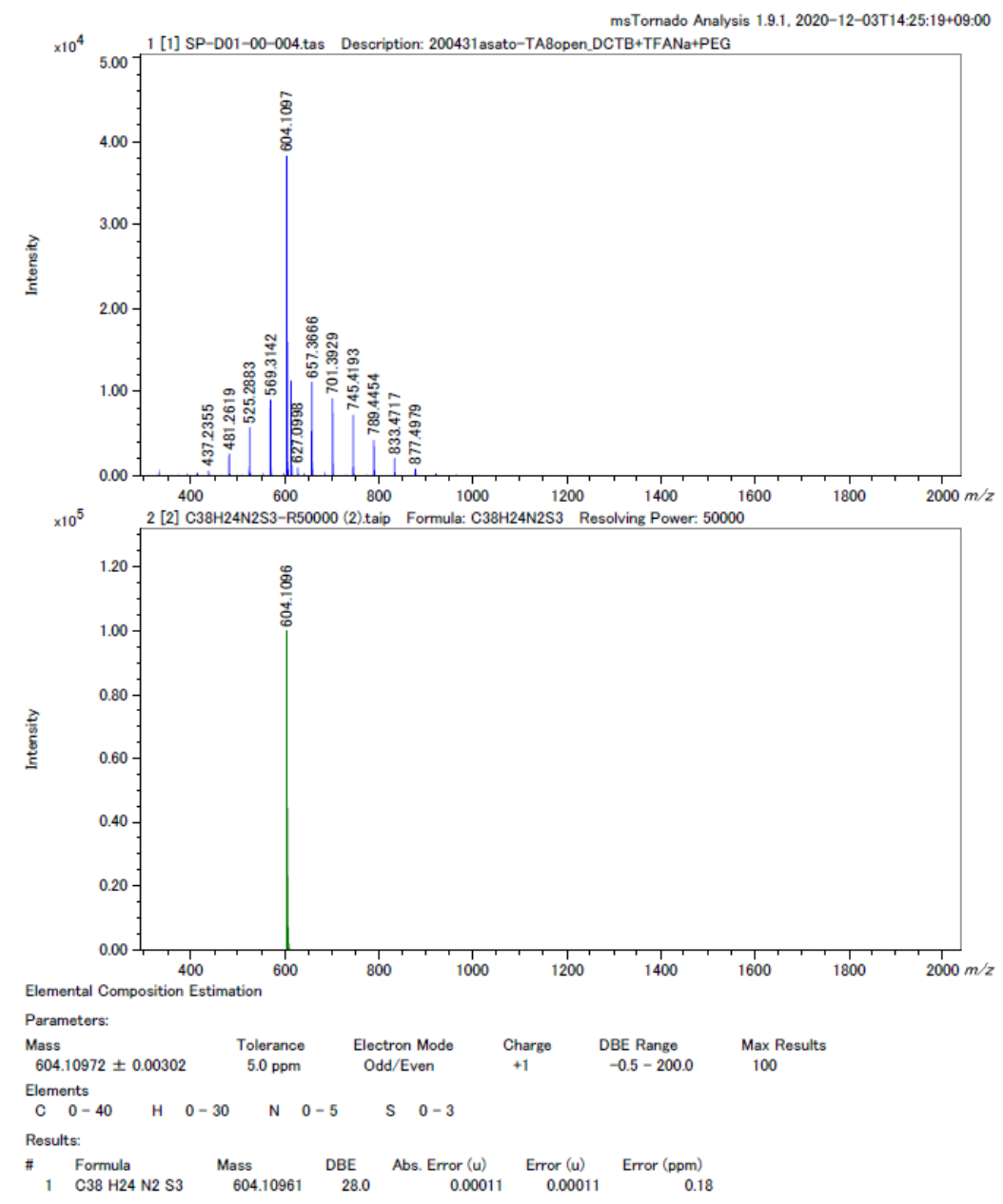

Figure S8. HR-MS data measurement (Top) and calculation result (bottom) of 4o. MALDI-SpiralTOF system with polyethylene glycol as internal standard. 


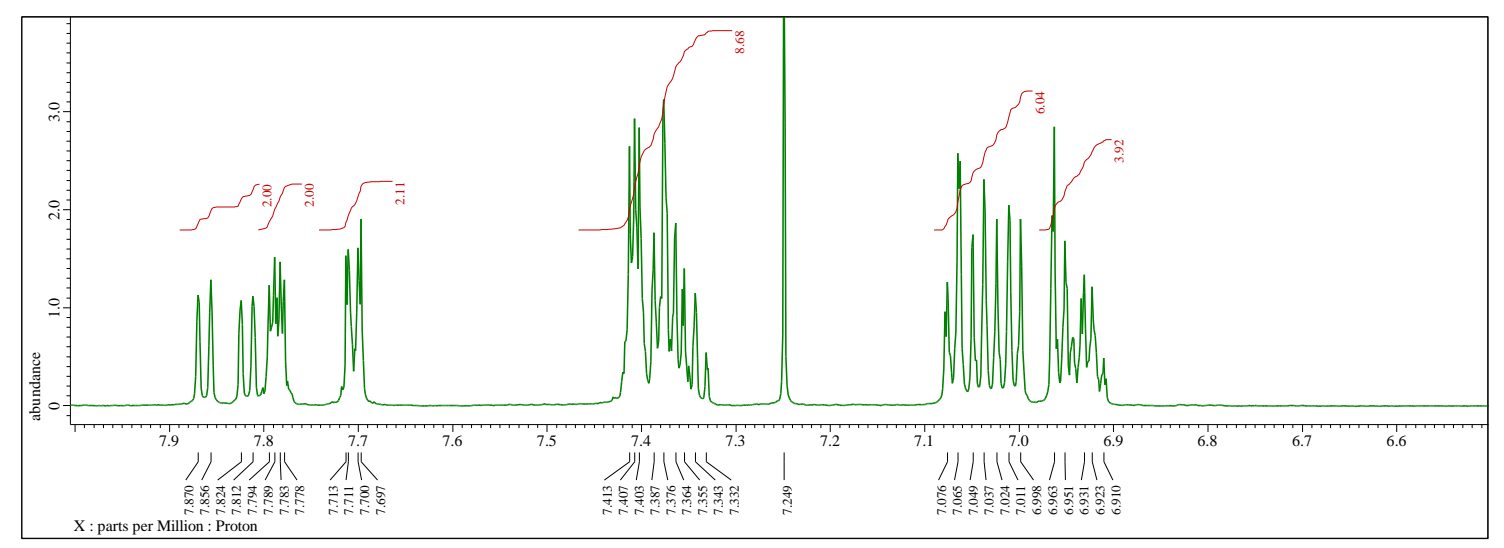

Figure S9. ${ }^{1} \mathrm{H}-\mathrm{NMR}$ spectrum of $40\left(600 \mathrm{MHz}, \mathrm{CDCl} 3, \mathrm{TMS}, 25^{\circ} \mathrm{C}\right)$.

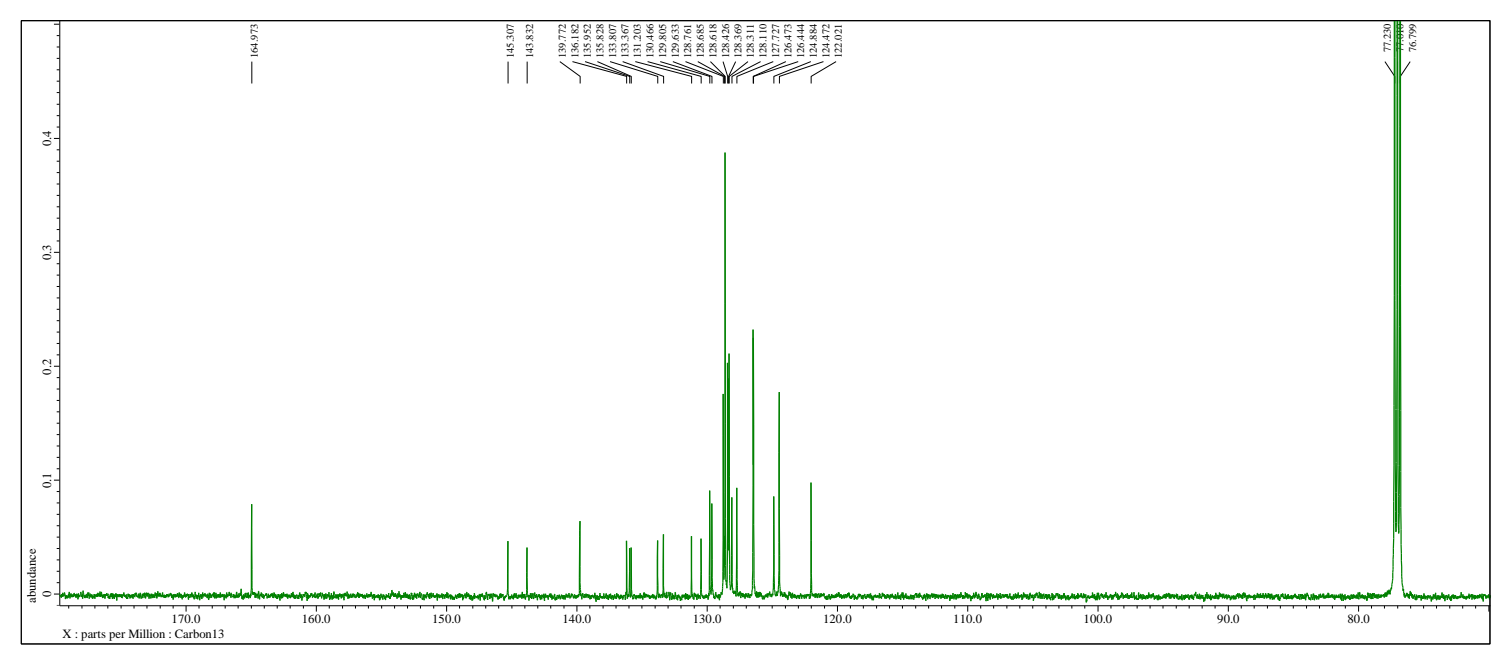

Figure S10. ${ }^{13} \mathrm{C} N M R$ spectrum of $40\left(151 \mathrm{MHz}, \mathrm{CDCl}_{3}, \mathrm{TMS}, 25^{\circ} \mathrm{C}\right)$.
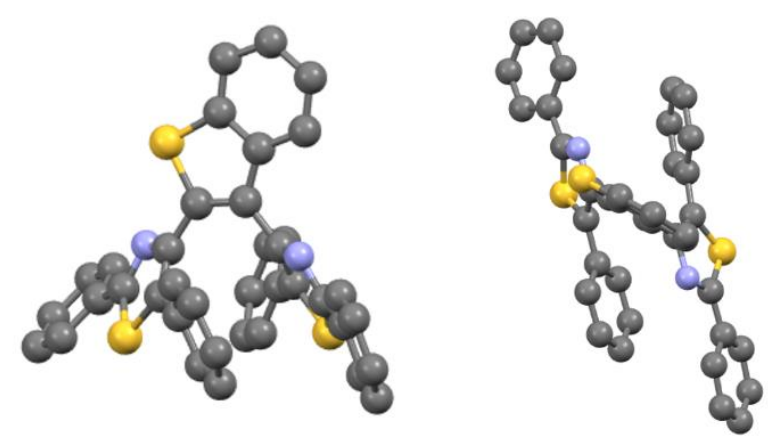

Figure S11. X-ray Structure of 40. Gray: carbon, yellow: sulfur, blue: nitrogen. 
Table S4. Crystallographic parameters and refinement details for 40 [CCDC 2051220].

\begin{tabular}{|c|c|}
\hline & 40 \\
\hline formula sum & $\mathrm{C}_{38} \mathrm{H}_{24} \mathrm{~N}_{2} \mathrm{~S}_{3}$ \\
\hline formula weight & 604.80 \\
\hline crystal system & triclinic \\
\hline space group & $P-1$ \\
\hline$a(\AA)$ & $8.46825(18)$ \\
\hline$b(\AA)$ & $12.1327(3)$ \\
\hline$c(\AA ̊)$ & 14.7821(3) \\
\hline$\alpha($ deg) & $106.138(8)$ \\
\hline$B(d e g)$ & $94.533(7)$ \\
\hline$\gamma($ deg $)$ & $91.905(7)$ \\
\hline$V\left(\AA^{3}\right)$ & 1451.92(8) \\
\hline$T(K)$ & 123.15 \\
\hline$Z$ & 2 \\
\hline $\begin{array}{l}\rho \text { calcd } \\
\left(g_{\mathrm{cm}^{-3}}\right)\end{array}$ & 1.383 \\
\hline$R 1[|>2 \sigma|)]$ & 0.0367 \\
\hline$w R 2[I>2 \sigma l)]$ & 0.0953 \\
\hline $\begin{array}{c}\text { Max Shift/Error } \\
\text { in Final Cycle }\end{array}$ & 0.001 \\
\hline
\end{tabular}




\section{DFT CALCULATIONS}

Formchk summary for 10.

Calculation Type $=$ FREQ. Calculation Method $=$ RB3LYP. Formula $\mathrm{C}_{27} \mathrm{H}_{19} \mathrm{NS}_{3}$.

Basis Set $=6-31 G(D)$. Charge $=0$. Spin $=$ Singlet. Solvation $=$ None .

Total Energy = -2289.6723 a.u. Sum of Electronic and Thermal Free Energies = -2289.349 a.u.

RMS Gradient Norm = 3.213e-06 a.u. Imaginary Freq = None.

Z-Matrix Cartesian coordinates.

\begin{tabular}{|c|c|c|c|c|c|c|c|c|c|}
\hline \multirow[t]{2}{*}{ Centre } & \multirow[t]{2}{*}{ Symbol } & \multicolumn{3}{|c|}{ Coordinates (Angstroms) } & \multirow[t]{2}{*}{ Centre } & \multirow[t]{2}{*}{ Symbol } & \multicolumn{3}{|c|}{ Coordinates (Angstroms) } \\
\hline & & $\mathrm{x}$ & $\mathrm{Y}$ & Z & & & $\mathrm{x}$ & $Y$ & Z \\
\hline 1 & $\mathrm{~N}$ & 1.89592 & 0.053676 & -0.31296 & 26 & C & -4.99188 & 2.413422 & 0.047192 \\
\hline 2 & C & 0.551801 & -0.23574 & -0.20749 & 27 & C & -4.73704 & 3.401081 & -0.89882 \\
\hline 3 & C & -0.24486 & 0.802856 & 0.245819 & 28 & C & -3.4408 & 3.583225 & -1.40911 \\
\hline 4 & $\mathrm{~S}$ & 0.76342 & 2.215609 & 0.513523 & 29 & C & -2.38672 & 2.787054 & -0.97822 \\
\hline 5 & C & 2.175933 & 1.285564 & 0.017245 & 30 & C & 1.607381 & -5.3271 & 0.800518 \\
\hline 6 & C & 0.103496 & -1.59605 & -0.57407 & 31 & C & 0.560066 & -5.2448 & -0.11122 \\
\hline 7 & C & 3.528617 & 1.857735 & -0.02897 & 32 & $\mathrm{H}$ & -2.04612 & -1.08067 & 3.326337 \\
\hline 8 & C & -1.67758 & 0.838459 & 0.570303 & 33 & $\mathrm{H}$ & -1.84671 & -2.07847 & 1.87754 \\
\hline 9 & C & 0.728034 & -2.79299 & -0.02985 & 34 & $\mathrm{H}$ & -0.56745 & -0.9576 & 2.351058 \\
\hline 10 & C & 0.131315 & -3.97835 & -0.51803 & 35 & $\mathrm{H}$ & -1.86914 & -1.30437 & -3.29306 \\
\hline 11 & $S$ & -1.1602 & -3.61136 & -1.64894 & 36 & $\mathrm{H}$ & -2.75261 & -0.82684 & -1.83608 \\
\hline 12 & $C$ & -0.90335 & -1.87814 & -1.4593 & 37 & $\mathrm{H}$ & -1.2927 & 0.050537 & -2.30417 \\
\hline 13 & $C$ & -2.27189 & -0.00245 & 1.47807 & 38 & $\mathrm{H}$ & 2.997048 & 3.797954 & 0.771322 \\
\hline 14 & $\mathrm{~S}$ & -3.9962 & 0.305648 & 1.645333 & 39 & $\mathrm{H}$ & 5.287892 & 4.695032 & 0.664314 \\
\hline 15 & $C$ & -3.92897 & 1.616096 & 0.479488 & 40 & $\mathrm{H}$ & 7.150169 & 3.28225 & -0.18727 \\
\hline 16 & $\mathrm{C}$ & -2.61503 & 1.787327 & -0.01491 & 41 & $\mathrm{H}$ & 6.688024 & 0.954453 & -0.93645 \\
\hline 17 & $C$ & -1.65156 & -1.08699 & 2.304192 & 42 & $\mathrm{H}$ & 4.372169 & 0.053383 & -0.83483 \\
\hline 18 & $C$ & -1.74999 & -0.94112 & -2.26604 & 43 & $\mathrm{H}$ & 2.271202 & -2.00765 & 1.262237 \\
\hline 19 & $C$ & 3.799172 & 3.169581 & 0.392421 & 44 & $\mathrm{H}$ & 3.035299 & -4.24464 & 2.005047 \\
\hline 20 & $C$ & 5.094814 & 3.67806 & 0.334251 & 45 & $\mathrm{H}$ & -5.99355 & 2.264878 & 0.440306 \\
\hline 21 & $C$ & 6.13996 & 2.884616 & -0.14294 & 46 & $\mathrm{H}$ & -5.54903 & 4.033586 & -1.24637 \\
\hline 22 & $\mathrm{C}$ & 5.879706 & 1.57727 & -0.56286 & 47 & $\mathrm{H}$ & -3.2602 & 4.355014 & -2.15211 \\
\hline 23 & C & 4.586906 & 1.065209 & -0.5086 & 48 & $\mathrm{H}$ & -1.39006 & 2.933119 & -1.38343 \\
\hline 24 & $C$ & 1.786211 & -2.90406 & 0.890716 & 49 & $\mathrm{H}$ & 1.958009 & -6.30184 & 1.128356 \\
\hline 25 & C & 2.215686 & -4.16134 & 1.296536 & 50 & $\mathrm{H}$ & 0.087322 & -6.14291 & -0.49852 \\
\hline
\end{tabular}


Formchk summary for $\mathbf{1 c}$.

Calculation Type $=$ FREQ. Calculation Method $=$ RB3LYP. Formula $\mathrm{C}_{27} \mathrm{H}_{19} \mathrm{NS}_{3}$.

Basis Set $=6-31 G(D)$. Charge $=0$. Spin $=$ Singlet. Solvation $=$ None .

Total Energy $=-2289.6485$ a.u. Sum of Electronic and Thermal Free Energies $=-2289.320$ a.u.

RMS Gradient Norm $=4.7895$ e- 05 a.u. Imaginary Freq $=$ None .

Z-Matrix Cartesian coordinates.

\begin{tabular}{|c|c|c|c|c|}
\hline \multirow[t]{2}{*}{ Centre } & \multirow[t]{2}{*}{ Symbol } & \multicolumn{3}{|c|}{ Coordinates (Angstroms) } \\
\hline & & $x$ & $\mathrm{Y}$ & Z \\
\hline 1 & $\mathrm{~N}$ & 2.028236 & 0.231744 & 0.04444 \\
\hline 2 & C & 0.645167 & 0.330222 & -0.01246 \\
\hline 3 & C & -0.05248 & -0.96687 & -0.01978 \\
\hline 4 & S & 1.162907 & -2.248 & -0.13492 \\
\hline 5 & C & 2.449944 & -0.98669 & -0.018 \\
\hline 6 & C & -0.05678 & 1.500613 & -0.10982 \\
\hline 7 & C & 3.865712 & -1.37104 & 0.004719 \\
\hline 8 & C & -1.40096 & -1.07191 & 0.099716 \\
\hline 9 & C & 0.409314 & 2.871789 & -0.06139 \\
\hline 10 & C & -0.65024 & 3.807268 & 0.02306 \\
\hline 11 & $\mathrm{~S}$ & -2.26008 & 3.068714 & 0.069444 \\
\hline 12 & C & -1.55992 & 1.395059 & -0.40482 \\
\hline 13 & C & -2.16634 & 0.230165 & 0.414212 \\
\hline 14 & $S$ & -3.94412 & -0.13888 & -0.05141 \\
\hline 15 & C & -3.64575 & -1.88533 & -0.00957 \\
\hline 16 & C & -2.27533 & -2.23247 & 0.061563 \\
\hline 17 & C & -2.11829 & 0.454662 & 1.944892 \\
\hline 18 & C & -1.73199 & 1.235599 & -1.93687 \\
\hline 19 & C & 4.273317 & -2.70483 & -0.16143 \\
\hline 20 & C & 5.625672 & -3.03901 & -0.13672 \\
\hline 21 & C & 6.589585 & -2.04808 & 0.05601 \\
\hline 22 & C & 6.193389 & -0.71757 & 0.223564 \\
\hline 23 & C & 4.844961 & -0.37901 & 0.198048 \\
\hline 24 & C & 1.733523 & 3.34545 & -0.07093 \\
\hline 25 & C & 1.981154 & 4.713307 & 0.000086 \\
\hline
\end{tabular}

\begin{tabular}{|c|c|c|c|c|}
\hline \multirow[t]{2}{*}{ Centre } & \multirow[t]{2}{*}{ Symbol } & \multicolumn{3}{|c|}{ Coordinates (Angstroms) } \\
\hline & & $x$ & Y & Z \\
\hline 26 & C & -4.63908 & -2.86047 & -0.06361 \\
\hline 27 & C & -4.26796 & -4.20702 & -0.05802 \\
\hline 28 & H & -2.48993 & -0.43921 & 2.452953 \\
\hline 29 & $\mathrm{H}$ & -1.09016 & 0.639204 & 2.273121 \\
\hline 30 & $\mathrm{H}$ & -2.73513 & 1.305583 & 2.240195 \\
\hline 31 & $\mathrm{H}$ & -1.21433 & 2.053688 & -2.44454 \\
\hline 32 & $\mathrm{H}$ & -1.30163 & 0.287564 & -2.27491 \\
\hline 33 & H & -2.78598 & 1.25976 & -2.22014 \\
\hline 34 & $\mathrm{H}$ & 3.535155 & -3.48693 & -0.31561 \\
\hline 35 & $\mathrm{H}$ & 5.925665 & -4.07471 & -0.26839 \\
\hline 36 & $\mathrm{H}$ & 7.643821 & -2.3101 & 0.076523 \\
\hline 37 & $\mathrm{H}$ & 6.939438 & 0.057494 & 0.375746 \\
\hline 38 & $\mathrm{H}$ & 4.527314 & 0.649162 & 0.330769 \\
\hline 39 & C & -2.91947 & -4.57085 & 0.011276 \\
\hline 40 & C & -1.92775 & -3.59342 & 0.071655 \\
\hline 41 & $\mathrm{H}$ & -5.68639 & -2.57724 & -0.11293 \\
\hline 42 & $\mathrm{H}$ & -5.03607 & -4.97405 & -0.10513 \\
\hline 43 & $\mathrm{H}$ & -2.64 & -5.62031 & 0.023563 \\
\hline 44 & H & -0.88721 & -3.89292 & 0.140689 \\
\hline 45 & C & 0.920507 & 5.624527 & 0.078392 \\
\hline 46 & C & -0.402 & 5.177332 & 0.086154 \\
\hline 47 & $\mathrm{H}$ & 2.545719 & 2.631548 & -0.13651 \\
\hline 48 & $\mathrm{H}$ & 3.005223 & 5.075571 & -0.01147 \\
\hline 49 & $\mathrm{H}$ & 1.123827 & 6.690711 & 0.131096 \\
\hline 50 & $\mathrm{H}$ & -1.22384 & 5.884911 & 0.144484 \\
\hline
\end{tabular}


Formchk summary for $\mathbf{2 o}$.

Calculation Type $=$ FREQ. Calculation Method $=$ RB3LYP. Formula $\mathrm{C}_{37} \mathrm{H}_{23} \mathrm{NS}_{3}$.

Basis Set $=6-31 G(D)$. Charge $=0$. Spin $=$ Singlet. Solvation $=$ None .

Total Energy $=-2673.143$ a.u. Sum of Electronic and Thermal Free Energies $=-2672.723$ a.u.

RMS Gradient Norm $=8.58$ e-07 a.u. Imaginary Freq $=$ None .

Z-Matrix Cartesian coordinates.

\begin{tabular}{|c|c|c|c|c|}
\hline \multirow[t]{2}{*}{ Centre } & \multirow[t]{2}{*}{ Symbol } & \multicolumn{3}{|c|}{ Coordinates (Angstroms) } \\
\hline & & $x$ & Y & Z \\
\hline 1 & $\mathrm{~N}$ & 1.613885 & -1.65366 & -0.19573 \\
\hline 2 & C & 0.41675 & -0.98489 & -0.06347 \\
\hline 3 & C & 0.457738 & 0.167796 & 0.707713 \\
\hline 4 & s & 2.098236 & 0.399149 & 1.279022 \\
\hline 5 & C & 2.594333 & -1.06064 & 0.435429 \\
\hline 6 & C & -0.78003 & -1.58709 & -0.69881 \\
\hline 7 & C & 3.978237 & -1.55431 & 0.439521 \\
\hline 8 & C & -0.6133 & 1.082274 & 1.14201 \\
\hline 9 & C & -1.26296 & -2.90409 & -0.3203 \\
\hline 10 & C & -2.46561 & -3.24913 & -0.97972 \\
\hline 11 & $S$ & -2.96389 & -1.98279 & -2.08371 \\
\hline 12 & C & -1.56535 & -0.99203 & -1.65198 \\
\hline 13 & C & -0.56084 & 2.457896 & 1.040074 \\
\hline 14 & S & -1.98563 & 3.23437 & 1.730937 \\
\hline 15 & C & -2.65909 & 1.692282 & 2.211299 \\
\hline 16 & C & -1.80708 & 0.625567 & 1.841205 \\
\hline 17 & C & 0.472087 & 3.33894 & 0.461388 \\
\hline 18 & C & -1.33588 & 0.240652 & -2.43205 \\
\hline 19 & C & 5.013786 & -0.86715 & 1.093407 \\
\hline 20 & C & 6.313877 & -1.3674 & 1.079629 \\
\hline 21 & C & 6.600896 & -2.56022 & 0.413002 \\
\hline 22 & C & 5.576547 & -3.24988 & -0.2415 \\
\hline 23 & C & 4.276193 & -2.75443 & -0.23068 \\
\hline 24 & C & -0.70905 & -3.81925 & 0.595011 \\
\hline 25 & C & -1.35071 & -5.02796 & 0.83097 \\
\hline 26 & C & -3.85735 & 1.482851 & 2.900925 \\
\hline 27 & C & -4.20383 & 0.181257 & 3.243386 \\
\hline 28 & c & -3.35585 & -0.89076 & 2.915485 \\
\hline
\end{tabular}

\begin{tabular}{|c|c|c|c|c|}
\hline \multirow[t]{2}{*}{ Centre } & \multirow[t]{2}{*}{ Symbol } & \multicolumn{3}{|c|}{ Coordinates (Angstroms) } \\
\hline & & $\mathrm{x}$ & Y & Z \\
\hline 33 & C & 1.844254 & 5.344607 & 0.603498 \\
\hline 34 & C & 2.415888 & 5.053046 & -0.63617 \\
\hline 35 & C & 2.012379 & 3.909478 & -1.32857 \\
\hline 36 & C & 1.048191 & 3.060905 & -0.78917 \\
\hline 37 & C & -0.1431 & 0.387855 & -3.16196 \\
\hline 38 & C & 0.053663 & 1.501816 & -3.97784 \\
\hline 39 & C & -0.93258 & 2.486356 & -4.07351 \\
\hline 40 & $\mathrm{H}$ & 4.807177 & 0.064775 & 1.613566 \\
\hline 41 & $\mathrm{H}$ & 7.103795 & -0.82332 & 1.590075 \\
\hline 42 & $\mathrm{H}$ & 7.615406 & -2.94922 & 0.403048 \\
\hline 43 & $\mathrm{H}$ & 5.792398 & -4.17854 & -0.76302 \\
\hline 44 & $\mathrm{H}$ & 3.473371 & -3.28062 & -0.73565 \\
\hline 45 & $\mathrm{H}$ & 0.225188 & -3.58167 & 1.093616 \\
\hline 46 & $\mathrm{H}$ & -0.91884 & -5.73671 & 1.532168 \\
\hline 47 & $\mathrm{H}$ & -4.49568 & 2.319501 & 3.170357 \\
\hline 48 & $\mathrm{H}$ & -5.1307 & -0.00523 & 3.77867 \\
\hline 49 & $\mathrm{H}$ & -3.63001 & -1.90124 & 3.20487 \\
\hline 50 & $\mathrm{H}$ & -1.52051 & -1.51378 & 1.995683 \\
\hline 51 & $\mathrm{H}$ & -3.03692 & -6.30019 & 0.367045 \\
\hline 52 & $\mathrm{H}$ & -4.04148 & -4.70912 & -1.25316 \\
\hline 53 & $\mathrm{H}$ & 0.452304 & 4.721549 & 2.120425 \\
\hline 54 & $\mathrm{H}$ & 2.151364 & 6.23086 & 1.152319 \\
\hline 55 & $\mathrm{H}$ & 3.1673 & 5.713486 & -1.06063 \\
\hline 56 & $\mathrm{H}$ & 2.441595 & 3.679309 & -2.30002 \\
\hline 57 & $\mathrm{H}$ & 0.724097 & 2.188359 & -1.34342 \\
\hline 58 & C & -2.11802 & 2.350813 & -3.34865 \\
\hline 59 & C & -2.32225 & 1.234322 & -2.53824 \\
\hline 60 & $\mathrm{H}$ & 0.614291 & -0.38794 & -3.09981 \\
\hline
\end{tabular}




\begin{tabular}{|lllll|}
\hline 29 & $C$ & -2.16881 & -0.678 & 2.228309 \\
30 & $C$ & -2.54961 & -5.34929 & 0.170046 \\
31 & $C$ & -3.11672 & -4.46305 & -0.73911 \\
32 & $C$ & 0.88075 & 4.497209 & 1.147638 \\
\hline
\end{tabular}

\begin{tabular}{|lllll|}
\hline 61 & $\mathrm{H}$ & 0.975042 & 1.594938 & -4.54694 \\
62 & $\mathrm{H}$ & -0.77888 & 3.353141 & -4.71043 \\
63 & $\mathrm{H}$ & -2.88705 & 3.115621 & -3.41343 \\
64 & $\mathrm{H}$ & -3.24521 & 1.131695 & -1.97455 \\
\hline
\end{tabular}


Formchk summary for $\mathbf{2 c}$.

Calculation Type $=$ FREQ. Calculation Method $=$ RB3LYP. Formula $\mathrm{C}_{37} \mathrm{H}_{23} \mathrm{NS}_{3}$.

Basis Set $=6-31 G(D)$. Charge $=0$. Spin $=$ Singlet. Solvation $=$ None .

Total Energy $=-2673.095$ a.u. Sum of Electronic and Thermal Free Energies $=-2672.672$ a.u.

RMS Gradient Norm $=8.904 \mathrm{e}-06$ a.u. Imaginary Freq $=$ None.

Z-Matrix Cartesian coordinates.

\begin{tabular}{|c|c|c|c|c|}
\hline \multirow[t]{2}{*}{ Centre } & \multirow[t]{2}{*}{ Symbol } & \multicolumn{3}{|c|}{ Coordinates (Angstroms) } \\
\hline & & $x$ & $\mathrm{Y}$ & Z \\
\hline 1 & $\mathrm{~N}$ & 2.44859 & 0.447308 & 0.108269 \\
\hline 2 & C & 1.069647 & 0.310868 & 0.023726 \\
\hline 3 & C & 0.608222 & -1.07889 & -0.10618 \\
\hline 4 & $S$ & 2.028852 & -2.11919 & -0.29915 \\
\hline 5 & C & 3.075924 & -0.67037 & -0.04518 \\
\hline 6 & C & 0.181904 & 1.349252 & -0.00874 \\
\hline 7 & C & 4.536366 & -0.80522 & -0.01595 \\
\hline 8 & C & -0.69846 & -1.42928 & -0.01516 \\
\hline 9 & C & 0.43677 & 2.768683 & 0.144034 \\
\hline 10 & C & -0.74876 & 3.513718 & 0.336778 \\
\hline 11 & $S$ & -2.2069 & 2.519597 & 0.390354 \\
\hline 12 & C & -1.28861 & 1.039195 & -0.33091 \\
\hline 13 & c & -1.70906 & -0.3242 & 0.359229 \\
\hline 14 & $S$ & -3.33604 & -0.99021 & -0.31971 \\
\hline 15 & c & -2.73058 & -2.64915 & -0.28908 \\
\hline 16 & C & -1.32785 & -2.73483 & -0.14435 \\
\hline 17 & C & -1.82877 & -0.22406 & 1.910106 \\
\hline 18 & C & -1.50001 & 1.044753 & -1.87735 \\
\hline 19 & C & 5.173645 & -2.01815 & -0.32505 \\
\hline 20 & C & 6.563115 & -2.1145 & -0.29176 \\
\hline 21 & C & 7.335003 & -1.00365 & 0.052504 \\
\hline 22 & C & 6.709268 & 0.207576 & 0.36344 \\
\hline 23 & C & 5.322912 & 0.309378 & 0.32983 \\
\hline 24 & C & 1.669063 & 3.444988 & 0.137466 \\
\hline 25 & c & 1.702034 & 4.825298 & 0.316018 \\
\hline 26 & C & -3.5183 & -3.78835 & -0.43565 \\
\hline 27 & C & -2.89908 & -5.0401 & -0.44758 \\
\hline 28 & C & -1.51233 & -5.14695 & -0.30375 \\
\hline
\end{tabular}

\begin{tabular}{|c|c|c|c|c|}
\hline \multirow[t]{2}{*}{ Centre } & \multirow[t]{2}{*}{ Symbol } & \multicolumn{3}{|c|}{ Coordinates (Angstroms) } \\
\hline & & $x$ & Y & Z \\
\hline 33 & C & -3.13919 & 0.314181 & 3.89589 \\
\hline 34 & C & -2.06327 & -0.01453 & 4.722034 \\
\hline 35 & C & -0.87474 & -0.45011 & 4.142377 \\
\hline 36 & C & -0.76013 & -0.55498 & 2.75397 \\
\hline 37 & C & -2.74459 & 1.389467 & -2.42957 \\
\hline 38 & C & -2.95444 & 1.385898 & -3.80605 \\
\hline 39 & C & -1.9182 & 1.043523 & -4.67616 \\
\hline 40 & C & -0.67547 & 0.706592 & -4.14681 \\
\hline 41 & C & -0.46925 & 0.708769 & -2.76504 \\
\hline 42 & $\mathrm{H}$ & 4.586065 & -2.8896 & -0.60024 \\
\hline 43 & $\mathrm{H}$ & 7.042393 & -3.05829 & -0.53578 \\
\hline 44 & $\mathrm{H}$ & 8.418447 & -1.08051 & 0.079725 \\
\hline 45 & $\mathrm{H}$ & 7.305572 & 1.074392 & 0.634554 \\
\hline 46 & $\mathrm{H}$ & 4.827269 & 1.242314 & 0.574302 \\
\hline 47 & H & 2.580118 & 2.878803 & -0.01191 \\
\hline 48 & $\mathrm{H}$ & 2.655393 & 5.345929 & 0.303622 \\
\hline 49 & $\mathrm{H}$ & -4.59583 & -3.70379 & -0.5412 \\
\hline 50 & H & -3.50374 & -5.93527 & -0.5645 \\
\hline 51 & $\mathrm{H}$ & -1.04027 & -6.1251 & -0.30217 \\
\hline 52 & H & 0.343368 & -4.10703 & -0.02107 \\
\hline 53 & $\mathrm{H}$ & 0.552899 & 6.624807 & 0.636864 \\
\hline 54 & $\mathrm{H}$ & -1.63801 & 5.455913 & 0.650542 \\
\hline 55 & $\mathrm{H}$ & -3.87991 & 0.44884 & 1.895451 \\
\hline 56 & $\mathrm{H}$ & -4.07824 & 0.651065 & 4.326936 \\
\hline 57 & H & -2.1547 & 0.062102 & 5.802226 \\
\hline 58 & $\mathrm{H}$ & -0.02687 & -0.71942 & 4.766766 \\
\hline 59 & H & 0.174067 & -0.91073 & 2.338816 \\
\hline 60 & $\mathrm{H}$ & -3.56122 & 1.679647 & -1.77823 \\
\hline
\end{tabular}




\begin{tabular}{|lllll|}
\hline 29 & $C$ & -0.72847 & -4.00402 & -0.15277 \\
30 & $C$ & 0.51654 & 5.547153 & 0.501714 \\
31 & $C$ & -0.71817 & 4.896133 & 0.509521 \\
32 & C & -3.02197 & 0.206849 & 2.512545 \\
\hline
\end{tabular}

\begin{tabular}{|lllll|}
\hline 61 & $\mathrm{H}$ & -3.93126 & 1.657952 & -4.19707 \\
62 & $\mathrm{H}$ & -2.07765 & 1.046903 & -5.75115 \\
63 & $\mathrm{H}$ & 0.148449 & 0.445214 & -4.80567 \\
64 & $\mathrm{H}$ & 0.514472 & 0.455819 & -2.39132 \\
\hline
\end{tabular}


Formchk summary for $\mathbf{3 o}$.

Calculation Type $=$ FREQ. Calculation Method $=$ RB3LYP. Formula $\mathrm{C}_{28} \mathrm{H}_{20} \mathrm{~N}_{2} \mathrm{~S}_{3}$.

Basis Set $=6-31 G(D)$. Charge $=0$. Spin $=$ Singlet. Solvation $=$ None .

Total Energy $=-2383.1306$ a.u. Sum of Electronic and Thermal Free Energies $=-2382.7900$ a.u.

RMS Gradient Norm = 5.328e-06 a.u. Imaginary Freq = None .

Z-Matrix Cartesian coordinates.

\begin{tabular}{|c|c|c|c|c|}
\hline \multirow[t]{2}{*}{ Centre } & \multirow[t]{2}{*}{ Symbol } & \multicolumn{3}{|c|}{ Coordinates (Angstroms) } \\
\hline & & $\mathrm{x}$ & Y & Z \\
\hline 1 & C & -0.19678 & 3.675063 & -0.24662 \\
\hline 2 & C & -0.60481 & 1.352853 & 0.089281 \\
\hline 3 & C & 0.7016 & 1.327745 & -0.33879 \\
\hline 4 & $S$ & 1.342338 & 2.943177 & -0.6547 \\
\hline 5 & C & -1.14919 & 2.698441 & 0.128114 \\
\hline 6 & C & -1.37901 & 0.176009 & 0.53948 \\
\hline 7 & C & -2.43877 & 3.119724 & 0.503536 \\
\hline 8 & C & 1.594815 & 0.190237 & -0.59529 \\
\hline 9 & $\mathrm{~N}$ & -2.62117 & -0.05731 & -0.01978 \\
\hline 10 & C & -3.20625 & -1.10958 & 0.483295 \\
\hline 11 & $S$ & -2.24065 & -1.91239 & 1.727138 \\
\hline 12 & C & -0.9923 & -0.71014 & 1.52171 \\
\hline 13 & C & 1.283964 & -0.93865 & -1.3251 \\
\hline 14 & $S$ & 2.685493 & -1.97501 & -1.385 \\
\hline 15 & C & 3.597627 & -0.78589 & -0.44628 \\
\hline 16 & N & 2.89278 & 0.263578 & -0.12774 \\
\hline 17 & C & 0.018922 & -1.30973 & -2.03538 \\
\hline 18 & C & 0.247614 & -0.73117 & 2.362111 \\
\hline 19 & C & 5.003706 & -0.98001 & -0.06881 \\
\hline 20 & C & -4.52827 & -1.59543 & 0.06567 \\
\hline 21 & C & -5.13115 & -2.71918 & 0.653388 \\
\hline 22 & C & -6.387 & -3.15068 & 0.23192 \\
\hline 23 & C & -7.06117 & -2.46727 & -0.78183 \\
\hline 24 & C & -6.46883 & -1.3474 & -1.37206 \\
\hline 25 & C & -5.21427 & -0.91302 & -0.95521 \\
\hline 26 & C & 5.656323 & 0.025491 & 0.666881 \\
\hline 27 & C & 6.988797 & -0.12728 & 1.03759 \\
\hline
\end{tabular}

\begin{tabular}{|c|c|c|c|c|}
\hline \multirow[t]{2}{*}{ Centre } & \multirow[t]{2}{*}{ Symbol } & \multicolumn{3}{|c|}{ Coordinates (Angstroms) } \\
\hline & & $\mathrm{x}$ & $\mathrm{Y}$ & z \\
\hline 28 & c & 7.692925 & -1.28164 & 0.683994 \\
\hline 29 & c & 7.05228 & -2.28481 & -0.04572 \\
\hline 30 & c & 5.718518 & -2.1364 & -0.4198 \\
\hline 31 & c & -2.74769 & 4.472803 & 0.492565 \\
\hline 32 & c & -1.78734 & 5.429314 & 0.116779 \\
\hline 33 & c & -0.50524 & 5.03949 & -0.25286 \\
\hline 34 & $\mathrm{H}$ & -3.18392 & 2.382222 & 0.781775 \\
\hline 35 & $\mathrm{H}$ & -3.74455 & 4.79807 & 0.777189 \\
\hline 36 & $\mathrm{H}$ & -0.54924 & -2.07098 & -1.48682 \\
\hline 37 & $\mathrm{H}$ & 0.228405 & -1.70787 & -3.03448 \\
\hline 38 & $\mathrm{H}$ & -0.62444 & -0.43302 & -2.14101 \\
\hline 39 & $\mathrm{H}$ & 0.933032 & -1.53181 & 2.057753 \\
\hline 40 & $\mathrm{H}$ & 0.009467 & -0.88334 & 3.420952 \\
\hline 41 & $\mathrm{H}$ & 0.782866 & 0.216197 & 2.262154 \\
\hline 42 & $\mathrm{H}$ & -4.62053 & -3.26083 & 1.445649 \\
\hline 43 & $\mathrm{H}$ & -6.8394 & -4.02189 & 0.697608 \\
\hline 44 & $\mathrm{H}$ & -8.04067 & -2.80461 & -1.10949 \\
\hline 45 & $\mathrm{H}$ & -6.98712 & -0.81025 & -2.16187 \\
\hline 46 & $\mathrm{H}$ & -4.74488 & -0.04595 & -1.40718 \\
\hline 47 & $\mathrm{H}$ & 5.100183 & 0.917046 & 0.935481 \\
\hline 48 & $\mathrm{H}$ & 7.48084 & 0.657997 & 1.605139 \\
\hline 49 & $\mathrm{H}$ & 8.733241 & -1.39841 & 0.975027 \\
\hline 50 & $\mathrm{H}$ & 7.591242 & -3.18583 & -0.32533 \\
\hline 51 & $\mathrm{H}$ & 5.234048 & -2.92678 & -0.98767 \\
\hline 52 & $\mathrm{H}$ & -2.04831 & 6.484086 & 0.11418 \\
\hline 53 & $\mathrm{H}$ & 0.239359 & 5.776123 & -0.54087 \\
\hline
\end{tabular}


Formchk summary for $\mathbf{3 c}$.

Calculation Type $=$ FREQ. Calculation Method $=$ RB3LYP. Formula $\mathrm{C}_{28} \mathrm{H}_{20} \mathrm{~N}_{2} \mathrm{~S}_{3}$.

Basis Set $=6-31 G(D)$. Charge $=0$. Spin $=$ Singlet. Solvation $=$ None .

Total Energy $=-2383.1088$ a.u. Sum of Electronic and Thermal Free Energies $=-2382.7617$ a.u.

RMS Gradient Norm $=6.413 \mathrm{e}-06$ a.u. Imaginary Freq $=$ None.

Z-Matrix Cartesian coordinates.

\begin{tabular}{|c|c|c|c|c|}
\hline \multirow[t]{2}{*}{ Centre } & \multirow[t]{2}{*}{ Symbol } & \multicolumn{3}{|c|}{ Coordinates (Angstroms) } \\
\hline & & $\mathrm{x}$ & Y & Z \\
\hline 1 & C & 0.381948 & 3.86854 & 0.011883 \\
\hline 2 & C & 0.720351 & 1.508214 & 0.024204 \\
\hline 3 & C & -0.73629 & 1.606632 & -0.02619 \\
\hline 4 & $S$ & -1.29719 & 3.28318 & 0.048506 \\
\hline 5 & C & 1.340048 & 2.830192 & -0.01168 \\
\hline 6 & C & 1.294307 & 0.267306 & 0.141797 \\
\hline 7 & C & 2.701682 & 3.164639 & -0.036 \\
\hline 8 & C & -1.53391 & 0.512415 & -0.1562 \\
\hline 9 & $\mathrm{~N}$ & 2.639396 & 0.009355 & 0.115147 \\
\hline 10 & C & 2.923084 & -1.25867 & 0.049626 \\
\hline 11 & $S$ & 1.520084 & -2.37762 & -0.02512 \\
\hline 12 & C & 0.398576 & -0.96142 & 0.42468 \\
\hline 13 & C & -0.88059 & -0.85558 & -0.43239 \\
\hline 14 & $\mathrm{~S}$ & -2.24923 & -2.03207 & 0.036763 \\
\hline 15 & C & -3.42131 & -0.6652 & -0.05688 \\
\hline 16 & N & -2.90378 & 0.523036 & -0.13551 \\
\hline 17 & C & -0.6307 & -0.98482 & -1.95379 \\
\hline 18 & C & 0.131356 & -1.02407 & 1.948729 \\
\hline 19 & C & -4.86413 & -0.90851 & -0.01486 \\
\hline 20 & C & 4.293381 & -1.77271 & 0.010892 \\
\hline 21 & C & 5.373035 & -0.86888 & -0.03358 \\
\hline 22 & C & 6.681127 & -1.33655 & -0.08008 \\
\hline 23 & C & 6.93921 & -2.71124 & -0.08308 \\
\hline 24 & C & 5.876807 & -3.61572 & -0.03799 \\
\hline 25 & C & 4.564086 & -3.15252 & 0.009761 \\
\hline 26 & C & -5.74577 & 0.189782 & 0.021558 \\
\hline 27 & C & -7.11973 & -0.01459 & 0.071534 \\
\hline
\end{tabular}

\begin{tabular}{|c|c|c|c|c|}
\hline \multirow[t]{2}{*}{ Centre } & \multirow[t]{2}{*}{ Symbol } & \multicolumn{3}{|c|}{ Coordinates (Angstroms) } \\
\hline & & $\mathrm{x}$ & Y & Z \\
\hline 28 & c & -7.64019 & -1.31299 & 0.086305 \\
\hline 29 & c & -6.77457 & -2.40753 & 0.049351 \\
\hline 30 & C & -5.39655 & -2.2092 & -0.0021 \\
\hline 31 & c & 3.084145 & 4.504375 & -0.03992 \\
\hline 32 & C & 2.121418 & 5.52028 & -0.01568 \\
\hline 33 & c & 0.760886 & 5.209007 & 0.012215 \\
\hline 34 & $\mathrm{H}$ & 3.438576 & 2.370311 & -0.05141 \\
\hline 35 & $\mathrm{H}$ & 4.139473 & 4.761258 & -0.06234 \\
\hline 36 & $\mathrm{H}$ & -0.30014 & -1.99139 & -2.21965 \\
\hline 37 & $\mathrm{H}$ & 0.132323 & -0.26994 & -2.27801 \\
\hline 38 & $\mathrm{H}$ & -1.55801 & -0.76941 & -2.49109 \\
\hline 39 & $\mathrm{H}$ & -0.38623 & -1.94606 & 2.22263 \\
\hline 40 & $\mathrm{H}$ & -0.48036 & -0.1738 & 2.265311 \\
\hline 41 & $\mathrm{H}$ & 1.083598 & -0.98591 & 2.484359 \\
\hline 42 & $\mathrm{H}$ & 5.162949 & 0.1947 & -0.0331 \\
\hline 43 & $\mathrm{H}$ & 7.504464 & -0.62837 & -0.11479 \\
\hline 44 & $\mathrm{H}$ & 7.962763 & -3.07399 & -0.11952 \\
\hline 45 & $\mathrm{H}$ & 6.069649 & -4.68481 & -0.03691 \\
\hline 46 & $\mathrm{H}$ & 3.74675 & -3.86631 & 0.05653 \\
\hline 47 & $\mathrm{H}$ & -5.32905 & 1.190908 & 0.012175 \\
\hline 48 & $\mathrm{H}$ & -7.78963 & 0.840429 & 0.100045 \\
\hline 49 & $\mathrm{H}$ & -8.71478 & -1.46935 & 0.125571 \\
\hline 50 & $\mathrm{H}$ & -7.17213 & -3.41854 & 0.05739 \\
\hline 51 & $\mathrm{H}$ & -4.73293 & -3.06822 & -0.0429 \\
\hline 52 & $\mathrm{H}$ & 2.430988 & 6.561853 & -0.01896 \\
\hline 53 & $\mathrm{H}$ & 0.013552 & 5.996966 & 0.031957 \\
\hline
\end{tabular}


Formchk summary for $\mathbf{4 o}$.

Calculation Type $=$ FREQ. Calculation Method $=$ RB3LYP. Formula $\mathrm{C}_{38} \mathrm{H}_{24} \mathrm{~N}_{2} \mathrm{~S}_{3}$.

Basis Set $=6-31 G(D)$. Charge $=0$. Spin $=$ Singlet. Solvation $=$ None.

Total Energy $=-2766.6026$ a.u. Sum of Electronic and Thermal Free Energies $=-2766.1657$ a.u.

RMS Gradient Norm $=1.1021 \mathrm{e}-05$ a.u. Imaginary Freq $=$ None.

Z-Matrix Cartesian coordinates.

\begin{tabular}{|c|c|c|c|c|}
\hline \multirow[t]{2}{*}{ Centre } & \multirow[t]{2}{*}{ Symbol } & \multicolumn{3}{|c|}{ Coordinates (Angstroms) } \\
\hline & & $x$ & Y & Z \\
\hline 1 & C & -1.09287 & 3.012052 & -0.11906 \\
\hline 2 & C & -0.58797 & 1.652205 & -0.07966 \\
\hline 3 & C & 0.613577 & 1.519646 & -0.73114 \\
\hline 4 & s & 1.213731 & 3.055065 & -1.36379 \\
\hline 5 & C & -0.21048 & 3.895812 & -0.78457 \\
\hline 6 & C & -1.30353 & 0.567285 & 0.627608 \\
\hline 7 & C & 1.446295 & 0.328546 & -0.95362 \\
\hline 8 & $\mathrm{~N}$ & -2.60982 & 0.311766 & 0.275358 \\
\hline 9 & C & -3.12781 & -0.67952 & 0.950152 \\
\hline 10 & $\mathrm{~S}$ & -2.01314 & -1.38272 & 2.120614 \\
\hline 11 & C & -0.78902 & -0.23087 & 1.634448 \\
\hline 12 & C & 1.028684 & -0.88877 & -1.46527 \\
\hline 13 & S & 2.394042 & -1.98192 & -1.49003 \\
\hline 14 & C & 3.422621 & -0.70234 & -0.84585 \\
\hline 15 & $\mathrm{~N}$ & 2.783277 & 0.416111 & -0.63709 \\
\hline 16 & C & -0.2712 & -1.31364 & -2.00881 \\
\hline 17 & C & 0.515623 & -0.19752 & 2.315283 \\
\hline 18 & C & -0.78602 & -2.59246 & -1.73202 \\
\hline 19 & C & -2.00232 & -3.0027 & -2.27481 \\
\hline 20 & C & -2.72595 & -2.14443 & -3.10421 \\
\hline 21 & C & -2.22154 & -0.874 & -3.39022 \\
\hline 22 & C & -1.00478 & -0.4615 & -2.85285 \\
\hline 23 & C & 1.190107 & -1.38759 & 2.640936 \\
\hline 24 & C & 2.415897 & -1.35351 & 3.303287 \\
\hline 25 & C & 2.991516 & -0.1309 & 3.652281 \\
\hline 26 & C & 2.328472 & 1.057481 & 3.33871 \\
\hline 27 & C & 1.100859 & 1.027585 & 2.681563 \\
\hline 28 & c & -4.49825 & -1.17194 & 0.760695 \\
\hline
\end{tabular}

\begin{tabular}{|c|c|c|c|c|}
\hline \multirow[t]{2}{*}{ Centre } & \multirow[t]{2}{*}{ Symbol } & \multicolumn{3}{|c|}{ Coordinates (Angstroms) } \\
\hline & & $x$ & Y & Z \\
\hline 35 & C & 7.590648 & -1.18424 & -0.01815 \\
\hline 36 & C & -6.35461 & -2.61935 & 1.361333 \\
\hline 37 & C & -5.04896 & -2.17966 & 1.568793 \\
\hline 38 & C & 6.878733 & -2.23132 & -0.60659 \\
\hline 39 & C & 5.520522 & -2.08788 & -0.88146 \\
\hline 40 & C & -2.28463 & 3.528204 & 0.424877 \\
\hline 41 & $\mathrm{H}$ & -0.23542 & -3.2601 & -1.07489 \\
\hline 42 & $\mathrm{H}$ & -2.3872 & -3.99226 & -2.04398 \\
\hline 43 & $\mathrm{H}$ & -3.67559 & -2.46348 & -3.52463 \\
\hline 44 & $\mathrm{H}$ & -2.77358 & -0.20175 & -4.04143 \\
\hline 45 & $\mathrm{H}$ & -0.6107 & 0.52034 & -3.09358 \\
\hline 46 & $\mathrm{H}$ & 0.756537 & -2.3428 & 2.357419 \\
\hline 47 & $\mathrm{H}$ & 2.924212 & -2.28413 & 3.540678 \\
\hline 48 & $\mathrm{H}$ & 3.948718 & -0.10433 & 4.16566 \\
\hline 49 & $\mathrm{H}$ & 2.764385 & 2.014153 & 3.613461 \\
\hline 50 & H & 0.582996 & 1.954463 & 2.458276 \\
\hline 51 & $\mathrm{H}$ & -4.85559 & 0.161486 & -0.88445 \\
\hline 52 & $\mathrm{H}$ & -7.19089 & -0.6132 & -1.25475 \\
\hline 53 & $\mathrm{H}$ & -8.15071 & -2.40101 & 0.186353 \\
\hline 54 & $\mathrm{H}$ & 5.052919 & 1.074646 & 0.267288 \\
\hline 55 & H & 7.48053 & 0.82756 & 0.753756 \\
\hline 56 & H & 8.650319 & -1.29669 & 0.194352 \\
\hline 57 & H & -6.76584 & -3.39915 & 1.996518 \\
\hline 58 & $\mathrm{H}$ & -4.45915 & -2.62135 & 2.368111 \\
\hline 59 & H & 7.381701 & -3.16171 & -0.85557 \\
\hline 60 & $\mathrm{H}$ & 4.981671 & -2.90998 & -1.34594 \\
\hline 61 & C & -2.56855 & 4.880334 & 0.292823 \\
\hline 62 & C & -1.6793 & 5.743109 & -0.3732 \\
\hline
\end{tabular}




\begin{tabular}{|lllll|}
\hline 29 & $C$ & 4.851898 & -0.89333 & -0.56908 \\
30 & C & -5.28759 & -0.61333 & -0.26076 \\
31 & C & -6.59202 & -1.05417 & -0.46222 \\
32 & C & -7.13193 & -2.05854 & 0.34611 \\
33 & C & 5.575825 & 0.156007 & 0.025003 \\
34 & $C$ & 6.932859 & 0.008535 & 0.295153 \\
\hline
\end{tabular}

\begin{tabular}{|ccccc|}
\hline 63 & $\mathrm{C}$ & -0.49395 & 5.259315 & -0.9151 \\
64 & $\mathrm{H}$ & -2.97835 & 2.861309 & 0.925638 \\
65 & $\mathrm{H}$ & -3.49072 & 5.278012 & 0.70755 \\
66 & $\mathrm{H}$ & -1.91976 & 6.798585 & -0.467 \\
67 & $\mathrm{H}$ & 0.195761 & 5.923683 & -1.42791 \\
\hline
\end{tabular}


Formchk summary for $\mathbf{4 c}$.

Calculation Type $=$ FREQ. Calculation Method $=$ RB3LYP. Formula $\mathrm{C}_{38} \mathrm{H}_{24} \mathrm{~N}_{2} \mathrm{~S}_{3}$.

Basis Set $=6-31 G(D)$. Charge $=0$. Spin $=$ Singlet. Solvation $=$ None .

Total Energy $=-2766.5585$ a.u. Sum of Electronic and Thermal Free Energies $=-2766.1167$ a.u.

RMS Gradient Norm $=3.826 \mathrm{e}-06$ a.u. Imaginary Freq $=$ None .

Z-Matrix Cartesian coordinates.

\begin{tabular}{|c|c|c|c|c|}
\hline \multirow[t]{2}{*}{ Centre } & \multirow[t]{2}{*}{ Symbol } & \multicolumn{3}{|c|}{ Coordinates (Angstroms) } \\
\hline & & $x$ & Y & Z \\
\hline 1 & C & 1.423664 & 3.071184 & -0.03765 \\
\hline 2 & C & 0.792447 & 1.753974 & 0.013163 \\
\hline 3 & C & -0.65993 & 1.863651 & -0.02729 \\
\hline 4 & s & -1.21051 & 3.543711 & 0.047735 \\
\hline 5 & C & 0.472948 & 4.116708 & -0.00969 \\
\hline 6 & C & 1.356494 & 0.511592 & 0.146983 \\
\hline 7 & C & -1.46908 & 0.780622 & -0.16254 \\
\hline 8 & $\mathrm{~N}$ & 2.703557 & 0.267296 & 0.11948 \\
\hline 9 & C & 2.997535 & -0.99553 & 0.031803 \\
\hline 10 & S & 1.609351 & -2.11272 & -0.08966 \\
\hline 11 & C & 0.465792 & -0.72502 & 0.434127 \\
\hline 12 & C & -0.8513 & -0.60847 & -0.42593 \\
\hline 13 & S & -2.25923 & -1.71752 & 0.128491 \\
\hline 14 & C & -3.38907 & -0.33367 & -0.03147 \\
\hline 15 & $\mathrm{~N}$ & -2.83812 & 0.834794 & -0.14493 \\
\hline 16 & C & -0.62592 & -0.83618 & -1.94599 \\
\hline 17 & C & 0.204419 & -0.86959 & 1.960216 \\
\hline 18 & C & -0.60125 & -2.13753 & -2.47264 \\
\hline 19 & C & -0.38988 & -2.36425 & -3.82974 \\
\hline 20 & C & -0.20457 & -1.28931 & -4.70164 \\
\hline 21 & C & -0.23594 & 0.007548 & -4.19589 \\
\hline 22 & C & -0.4454 & 0.232228 & -2.83262 \\
\hline 23 & C & 0.250383 & 0.227998 & 2.828107 \\
\hline 24 & C & 0.004843 & 0.074742 & 4.195219 \\
\hline 25 & C & -0.28973 & -1.17885 & 4.724433 \\
\hline 26 & C & -0.33301 & -2.28365 & 3.871621 \\
\hline 27 & C & -0.08533 & -2.1286 & 2.510667 \\
\hline 28 & c & 4.372027 & -1.49955 & -0.00864 \\
\hline
\end{tabular}

\begin{tabular}{|c|c|c|c|c|}
\hline \multirow[t]{2}{*}{ Centre } & \multirow[t]{2}{*}{ Symbol } & \multicolumn{3}{|c|}{ Coordinates (Angstroms) } \\
\hline & & $x$ & Y & Z \\
\hline 35 & C & 7.024885 & -2.41658 & -0.10694 \\
\hline 36 & C & -6.79173 & -1.97746 & 0.090234 \\
\hline 37 & C & -5.40848 & -1.82001 & 0.037361 \\
\hline 38 & C & 5.969835 & -3.32961 & -0.06245 \\
\hline 39 & C & 4.653536 & -2.87695 & -0.01219 \\
\hline 40 & C & 2.78722 & 3.396293 & -0.07766 \\
\hline 41 & H & -0.7639 & -2.9874 & -1.81734 \\
\hline 42 & H & -0.37616 & -3.38368 & -4.20606 \\
\hline 43 & $\mathrm{H}$ & -0.04588 & -1.46328 & -5.76252 \\
\hline 44 & $\mathrm{H}$ & -0.10449 & 0.857744 & -4.85989 \\
\hline 45 & $\mathrm{H}$ & -0.47819 & 1.252529 & -2.47118 \\
\hline 46 & $\mathrm{H}$ & 0.488753 & 1.214182 & 2.450148 \\
\hline 47 & $\mathrm{H}$ & 0.051872 & 0.945767 & 4.843331 \\
\hline 48 & $\mathrm{H}$ & -0.47699 & -1.29787 & 5.788219 \\
\hline 49 & H & -0.55523 & -3.27181 & 4.265479 \\
\hline 50 & $\mathrm{H}$ & -0.10619 & -3.00521 & 1.871054 \\
\hline 51 & $\mathrm{H}$ & -5.24123 & 1.576304 & 0.016975 \\
\hline 52 & $\mathrm{H}$ & -7.71142 & 1.299092 & 0.10646 \\
\hline 53 & $\mathrm{H}$ & -8.70365 & -0.98217 & 0.155648 \\
\hline 54 & H & 5.225428 & 0.4747 & -0.04922 \\
\hline 55 & $\mathrm{H}$ & 7.57344 & -0.32928 & -0.13447 \\
\hline 56 & $\mathrm{H}$ & 8.051304 & -2.77104 & -0.14511 \\
\hline 57 & $\mathrm{H}$ & -7.21885 & -2.97625 & 0.107613 \\
\hline 58 & $\mathrm{H}$ & -4.76976 & -2.69788 & 0.002527 \\
\hline 59 & $\mathrm{H}$ & 6.171321 & -4.39712 & -0.06289 \\
\hline 60 & H & 3.841718 & -3.59673 & 0.037093 \\
\hline 61 & C & 3.17859 & 4.733551 & -0.09241 \\
\hline 62 & c & 2.223437 & 5.756183 & -0.0634 \\
\hline
\end{tabular}




\begin{tabular}{|lllll|}
\hline 29 & $C$ & -4.83867 & -0.53575 & 0.011953 \\
30 & $C$ & -5.68731 & 0.588076 & 0.036235 \\
31 & $C$ & -7.06676 & 0.424606 & 0.087303 \\
32 & $C$ & -7.62491 & -0.85774 & 0.115463 \\
33 & $C$ & 5.443943 & -0.58714 & -0.05206 \\
34 & $C$ & 6.755828 & -1.04412 & -0.10058 \\
\hline
\end{tabular}

\begin{tabular}{|lllll|}
\hline 63 & $\mathrm{C}$ & 0.861038 & 5.454356 & -0.02005 \\
64 & $\mathrm{H}$ & 3.517815 & 2.596612 & -0.09625 \\
65 & $\mathrm{H}$ & 4.235405 & 4.982812 & -0.12712 \\
66 & $\mathrm{H}$ & 2.540067 & 6.795578 & -0.07523 \\
67 & $\mathrm{H}$ & 0.119288 & 6.247491 & 0.003407 \\
\hline
\end{tabular}


Formchk summary for $\mathbf{5 0}$.

Calculation Type $=$ FREQ. Calculation Method $=$ RB3LYP. Formula $\mathrm{C}_{29} \mathrm{H}_{21} \mathrm{~N}_{3} \mathrm{~S}_{3}$.

Basis Set $=6-31 G(D)$. Charge $=0$. Spin $=$ Singlet. Solvation $=$ None .

Total Energy $=-2476.586$ a.u. Sum of Electronic and Thermal Free Energies $=-2476.2278$ a.u.

RMS Gradient Norm $=6.792 \mathrm{e}-06$ a.u. Imaginary Freq $=$ None.

Z-Matrix Cartesian coordinates.

\begin{tabular}{|c|c|c|c|c|}
\hline \multirow[t]{2}{*}{ Centre } & \multirow[t]{2}{*}{ Symbol } & \multicolumn{3}{|c|}{ Coordinates (Angstroms) } \\
\hline & & $x$ & Y & Z \\
\hline 1 & $\mathrm{~N}$ & -1.28791 & 1.96893 & 0.281625 \\
\hline 2 & C & -0.64969 & 0.755159 & 0.181031 \\
\hline 3 & C & 0.659314 & 0.804464 & -0.26096 \\
\hline 4 & s & 1.117488 & 2.473779 & -0.51061 \\
\hline 5 & C & -0.50973 & 2.967302 & -0.04164 \\
\hline 6 & C & -1.39491 & -0.45617 & 0.590275 \\
\hline 7 & C & -0.93286 & 4.374071 & -0.00615 \\
\hline 8 & C & 1.623349 & -0.26083 & -0.55313 \\
\hline 9 & $\mathrm{~N}$ & -2.62627 & -0.69476 & 0.016715 \\
\hline 10 & C & -3.19039 & -1.77193 & 0.485991 \\
\hline 11 & s & -2.21378 & -2.59268 & 1.713487 \\
\hline 12 & C & -0.98659 & -1.36344 & 1.541769 \\
\hline 13 & C & 1.375018 & -1.40006 & -1.29095 \\
\hline 14 & S & 2.839564 & -2.34292 & -1.38062 \\
\hline 15 & C & 3.686844 & -1.10605 & -0.44307 \\
\hline 16 & $\mathrm{~N}$ & 2.92054 & -0.10792 & -0.10182 \\
\hline 17 & C & 0.124681 & -1.84648 & -1.98375 \\
\hline 18 & C & 0.256498 & -1.38591 & 2.377831 \\
\hline 19 & C & 5.108192 & -1.21272 & -0.08844 \\
\hline 20 & C & -4.50196 & -2.27102 & 0.051324 \\
\hline 21 & C & -5.24399 & -1.51554 & -0.87432 \\
\hline 22 & C & -6.49 & -1.95966 & -1.30624 \\
\hline 23 & C & -7.01823 & -3.16083 & -0.82491 \\
\hline 24 & C & -6.28809 & -3.91664 & 0.094222 \\
\hline 25 & C & -5.0397 & -3.47669 & 0.529198 \\
\hline 26 & C & 5.702638 & -0.18177 & 0.661135 \\
\hline 27 & C & 7.047534 & -0.25254 & 1.011115 \\
\hline 28 & c & 7.822064 & -1.34913 & 0.622668 \\
\hline
\end{tabular}

\begin{tabular}{|c|c|c|c|c|}
\hline \multirow[t]{2}{*}{ Centre } & \multirow[t]{2}{*}{ Symbol } & \multicolumn{3}{|c|}{ Coordinates (Angstroms) } \\
\hline & & $x$ & Y & Z \\
\hline 29 & C & 7.239545 & -2.37712 & -0.12108 \\
\hline 30 & C & 5.893657 & -2.31059 & -0.47461 \\
\hline 31 & C & -2.25359 & 4.677404 & 0.370189 \\
\hline 32 & C & -2.68637 & 5.999284 & 0.412068 \\
\hline 33 & C & -1.81347 & 7.039468 & 0.081302 \\
\hline 34 & C & -0.50065 & 6.746746 & -0.29332 \\
\hline 35 & C & -0.06272 & 5.425006 & -0.33776 \\
\hline 36 & $\mathrm{H}$ & -0.57334 & -1.01117 & -2.07879 \\
\hline 37 & $\mathrm{H}$ & 0.343233 & -2.22984 & -2.98662 \\
\hline 38 & $\mathrm{H}$ & -0.38814 & -2.6413 & -1.42832 \\
\hline 39 & $\mathrm{H}$ & 0.948021 & -2.17556 & 2.058571 \\
\hline 40 & $\mathrm{H}$ & 0.782866 & -0.43197 & 2.29356 \\
\hline 41 & $\mathrm{H}$ & 0.023283 & -1.55754 & 3.434781 \\
\hline 42 & $\mathrm{H}$ & -4.82461 & -0.58388 & -1.23836 \\
\hline 43 & H & -7.05301 & -1.36519 & -2.02082 \\
\hline 44 & $\mathrm{H}$ & -7.99169 & -3.50496 & -1.16347 \\
\hline 45 & $\mathrm{H}$ & -6.68983 & -4.85217 & 0.473703 \\
\hline 46 & $\mathrm{H}$ & -4.48184 & -4.07852 & 1.242274 \\
\hline 47 & $\mathrm{H}$ & 5.092029 & 0.664155 & 0.957517 \\
\hline 48 & $\mathrm{H}$ & 7.494 & 0.551401 & 1.590089 \\
\hline 49 & H & 8.871877 & -1.40208 & 0.89771 \\
\hline 50 & H & 7.833498 & -3.23362 & -0.42783 \\
\hline 51 & H & 5.455299 & -3.11903 & -1.05437 \\
\hline 52 & $\mathrm{H}$ & -2.92083 & 3.861094 & 0.62457 \\
\hline 53 & H & -3.70959 & 6.219657 & 0.704366 \\
\hline 54 & $\mathrm{H}$ & -2.15399 & 8.070798 & 0.115061 \\
\hline 55 & $\mathrm{H}$ & 0.1852 & 7.548692 & -0.55228 \\
\hline 56 & $\mathrm{H}$ & 0.962276 & 5.213425 & -0.63175 \\
\hline
\end{tabular}


Formchk summary for $\mathbf{5 c}$.

Calculation Type $=$ FREQ. Calculation Method $=$ RB3LYP. Formula $\mathrm{C}_{29} \mathrm{H}_{21} \mathrm{~N}_{3} \mathrm{~S}_{3}$.

Basis Set $=6-31 G(D)$. Charge $=0$. Spin $=$ Singlet. Solvation $=$ None .

Total Energy $=-2476.5602$ a.u. Sum of Electronic and Thermal Free Energies $=-2476.1947$ a.u.

RMS Gradient Norm $=4.813 \mathrm{e}-06$ a.u. Imaginary Freq $=$ None.

Z-Matrix Cartesian coordinates.

\begin{tabular}{|c|c|c|c|c|}
\hline \multirow[t]{2}{*}{ Centre } & \multirow[t]{2}{*}{ Symbol } & \multicolumn{3}{|c|}{ Coordinates (Angstroms) } \\
\hline & & $x$ & $\mathrm{Y}$ & z \\
\hline 1 & $\mathrm{~N}$ & -1.65 & 1.981445 & -0.00334 \\
\hline 2 & C & -0.82825 & 0.870278 & 0.031214 \\
\hline 3 & C & 0.600624 & 1.174491 & -0.02374 \\
\hline 4 & $S$ & 0.822926 & 2.918743 & 0.037056 \\
\hline 5 & C & -0.97986 & 3.087514 & 0.004433 \\
\hline 6 & C & -1.2431 & -0.43432 & 0.147924 \\
\hline 7 & C & -1.60513 & 4.414406 & -0.00186 \\
\hline 8 & C & 1.543737 & 0.203529 & -0.15022 \\
\hline 9 & $\mathrm{~N}$ & -2.53764 & -0.86307 & 0.117267 \\
\hline 10 & C & -2.64664 & -2.15715 & 0.048904 \\
\hline 11 & $S$ & -1.10283 & -3.08028 & -0.02406 \\
\hline 12 & C & -0.18427 & -1.52452 & 0.429435 \\
\hline 13 & c & 1.072628 & -1.24155 & -0.42442 \\
\hline 14 & $S$ & 2.584961 & -2.22587 & 0.045488 \\
\hline 15 & C & 3.567355 & -0.71864 & -0.05309 \\
\hline 16 & $\mathrm{~N}$ & 2.900045 & 0.393193 & -0.13203 \\
\hline 17 & C & 0.844643 & -1.40046 & -1.94667 \\
\hline 18 & C & 0.087224 & -1.55216 & 1.954114 \\
\hline 19 & C & 5.029777 & -0.77124 & -0.01449 \\
\hline 20 & C & -3.93764 & -2.84396 & 0.004377 \\
\hline 21 & C & -4.0334 & -4.24645 & 0.00076 \\
\hline 22 & C & -5.27864 & -4.86851 & -0.05208 \\
\hline 23 & C & -6.44454 & -4.10196 & -0.10015 \\
\hline 24 & C & -6.35933 & -2.70562 & -0.09511 \\
\hline 25 & C & -5.11991 & -2.07869 & -0.04341 \\
\hline 26 & C & 5.76051 & 0.432762 & 0.02048 \\
\hline 27 & C & 7.149506 & 0.409694 & 0.067093 \\
\hline 28 & C & 7.835123 & -0.8096 & 0.079825 \\
\hline
\end{tabular}

\begin{tabular}{|c|c|c|c|c|}
\hline \multirow[t]{2}{*}{ Centre } & \multirow[t]{2}{*}{ Symbol } & \multicolumn{3}{|c|}{ Coordinates (Angstroms) } \\
\hline & & $x$ & Y & Z \\
\hline 29 & C & 7.11982 & -2.00785 & 0.04431 \\
\hline 30 & C & 5.72761 & -1.99126 & -0.00368 \\
\hline 31 & C & -0.84469 & 5.59491 & -0.01756 \\
\hline 32 & C & -1.47256 & 6.838751 & -0.02193 \\
\hline 33 & C & -2.866 & 6.92081 & -0.01185 \\
\hline 34 & C & -3.63095 & 5.750173 & 0.002921 \\
\hline 35 & C & -3.00966 & 4.506202 & 0.008278 \\
\hline 36 & H & 1.736117 & -1.06431 & -2.48273 \\
\hline 37 & $\mathrm{H}$ & 0.649485 & -2.44152 & -2.21309 \\
\hline 38 & $\mathrm{H}$ & -0.0062 & -0.79292 & -2.27138 \\
\hline 39 & $\mathrm{H}$ & 0.724045 & -2.39616 & 2.228155 \\
\hline 40 & $\mathrm{H}$ & 0.578266 & -0.62754 & 2.273168 \\
\hline 41 & $\mathrm{H}$ & -0.86235 & -1.64347 & 2.487839 \\
\hline 42 & $\mathrm{H}$ & -3.13334 & -4.85272 & 0.04957 \\
\hline 43 & H & -5.33814 & -5.95328 & -0.05279 \\
\hline 44 & $\mathrm{H}$ & -7.4151 & -4.58892 & -0.14071 \\
\hline 45 & $\mathrm{H}$ & -7.26433 & -2.1054 & -0.13248 \\
\hline 46 & H & -5.03745 & -0.99737 & -0.04138 \\
\hline 47 & $\mathrm{H}$ & 5.216488 & 1.370821 & 0.012681 \\
\hline 48 & H & 7.702118 & 1.34485 & 0.094517 \\
\hline 49 & $\mathrm{H}$ & 8.921008 & -0.82426 & 0.116401 \\
\hline 50 & $\mathrm{H}$ & 7.646089 & -2.95823 & 0.050785 \\
\hline 51 & $\mathrm{H}$ & 5.181912 & -2.92966 & -0.04339 \\
\hline 52 & $\mathrm{H}$ & 0.240701 & 5.546005 & -0.02936 \\
\hline 53 & H & -0.87211 & 7.744009 & -0.03435 \\
\hline 54 & $\mathrm{H}$ & -3.35431 & 7.89152 & -0.01561 \\
\hline 55 & H & -4.71589 & 5.809256 & 0.011052 \\
\hline 56 & $\mathrm{H}$ & -3.58883 & 3.589382 & 0.021152 \\
\hline
\end{tabular}


Formchk summary for $\mathbf{6 o}$.

Calculation Type $=$ FREQ.$\quad$ Calculation Method $=$ RB3LYP. Formula $\mathrm{C}_{39} \mathrm{H}_{25} \mathrm{~N}_{3} \mathrm{~S}_{3}$.

Basis Set $=6-31 G(D)$. Charge $=0$. Spin $=$ Singlet. Solvation $=$ None .

Total Energy $=-2860.0583$ a.u. Sum of Electronic and Thermal Free Energies $=-2859.6037$ a.u.

RMS Gradient Norm $=4.501 \mathrm{e}-06$ a.u. Imaginary Freq $=$ None .

Z-Matrix Cartesian coordinates.

\begin{tabular}{|c|c|c|c|c|}
\hline \multirow[t]{2}{*}{ Centre } & \multirow[t]{2}{*}{ Symbol } & \multicolumn{3}{|c|}{ Coordinates (Angstroms) } \\
\hline & & $x$ & Y & Z \\
\hline 1 & $N$ & -1.18862 & 2.432137 & 0.313431 \\
\hline 2 & C & -0.62943 & 1.186594 & 0.157436 \\
\hline 3 & C & 0.571354 & 1.153011 & -0.52445 \\
\hline 4 & S & 1.037033 & 2.784141 & -0.9442 \\
\hline 5 & C & -0.44927 & 3.377942 & -0.20467 \\
\hline 6 & C & -1.3407 & 0.025383 & 0.740575 \\
\hline 7 & C & -0.80565 & 4.80263 & -0.16898 \\
\hline 8 & C & 1.452176 & 0.034 & -0.87863 \\
\hline 9 & $\mathrm{~N}$ & -2.63025 & -0.21558 & 0.333284 \\
\hline 10 & C & -3.14209 & -1.27317 & 0.901724 \\
\hline 11 & S & -2.03878 & -2.06342 & 2.030986 \\
\hline 12 & C & -0.82651 & -0.85406 & 1.674407 \\
\hline 13 & C & 1.07863 & -1.15147 & -1.48967 \\
\hline 14 & S & 2.49012 & -2.17471 & -1.63142 \\
\hline 15 & C & 3.473837 & -0.91092 & -0.8932 \\
\hline 16 & $\mathrm{~N}$ & 2.790308 & 0.154466 & -0.57496 \\
\hline 17 & C & -0.21168 & -1.58565 & -2.04821 \\
\hline 18 & C & 0.46874 & -0.85629 & 2.373265 \\
\hline 19 & C & -2.03087 & 5.183023 & 0.40766 \\
\hline 20 & C & -2.3985 & 6.524252 & 0.456264 \\
\hline 21 & C & -1.55477 & 7.507861 & -0.06719 \\
\hline 22 & C & -0.33674 & 7.138626 & -0.64138 \\
\hline 23 & C & 0.03596 & 5.797308 & -0.69276 \\
\hline 24 & C & -0.66553 & -2.90511 & -1.87485 \\
\hline 25 & C & -1.8733 & -3.3208 & -2.43214 \\
\hline 26 & C & -2.64914 & -2.42757 & -3.17272 \\
\hline 27 & C & -2.20547 & -1.11633 & -3.35593 \\
\hline 28 & C & -0.99721 & -0.69802 & -2.80425 \\
\hline
\end{tabular}

\begin{tabular}{|c|c|c|c|c|}
\hline \multirow[t]{2}{*}{ Centre } & \multirow[t]{2}{*}{ Symbol } & \multicolumn{3}{|c|}{ Coordinates (Angstroms) } \\
\hline & & $x$ & Y & Z \\
\hline 36 & C & -5.31261 & -1.07178 & -0.27275 \\
\hline 37 & C & -6.60472 & -1.51266 & -0.54192 \\
\hline 38 & C & -7.10855 & -2.65379 & 0.088754 \\
\hline 39 & C & 5.608617 & -0.02906 & 0.000471 \\
\hline 40 & C & 6.976155 & -0.13828 & 0.233188 \\
\hline 41 & C & 7.675622 & -1.27434 & -0.18346 \\
\hline 42 & C & -6.30706 & -3.35226 & 0.993796 \\
\hline 43 & C & -5.01299 & -2.91438 & 1.267378 \\
\hline 44 & C & 6.994896 & -2.30308 & -0.8375 \\
\hline 45 & C & 5.626216 & -2.19761 & -1.07489 \\
\hline 46 & $\mathrm{H}$ & -2.67699 & 4.410228 & 0.809702 \\
\hline 47 & $\mathrm{H}$ & -3.3482 & 6.804069 & 0.904341 \\
\hline 48 & $\mathrm{H}$ & -1.84444 & 8.554417 & -0.02802 \\
\hline 49 & H & 0.326154 & 7.896132 & -1.05048 \\
\hline 50 & H & 0.988266 & 5.526157 & -1.1416 \\
\hline 51 & $\mathrm{H}$ & -0.07444 & -3.60114 & -1.28587 \\
\hline 52 & H & -2.21118 & -4.3424 & -2.28123 \\
\hline 53 & $\mathrm{H}$ & -3.59295 & -2.75069 & -3.60284 \\
\hline 54 & H & -2.79919 & -0.41566 & -3.93651 \\
\hline 55 & H & -0.65063 & 0.317478 & -2.96539 \\
\hline 56 & $\mathrm{H}$ & 0.469591 & 1.270718 & 2.734968 \\
\hline 57 & $\mathrm{H}$ & 2.642951 & 1.275614 & 3.910504 \\
\hline 58 & $\mathrm{H}$ & 3.881983 & -0.85405 & 4.261316 \\
\hline 59 & $\mathrm{H}$ & 2.924429 & -2.98804 & 3.412534 \\
\hline 60 & $\mathrm{H}$ & 0.768171 & -2.98759 & 2.20516 \\
\hline 61 & $\mathrm{H}$ & -4.90939 & -0.1883 & -0.7553 \\
\hline 62 & $\mathrm{H}$ & -7.22315 & -0.96292 & -1.24661 \\
\hline 63 & $\mathrm{H}$ & -8.11819 & -2.99535 & -0.12279 \\
\hline
\end{tabular}




\begin{tabular}{|lllll|}
\hline 29 & $\mathrm{C}$ & 1.01538 & 0.342005 & 2.866359 \\
30 & $\mathrm{C}$ & 2.236767 & 0.339897 & 3.536028 \\
31 & $\mathrm{C}$ & 2.930396 & -0.85536 & 3.736881 \\
32 & $\mathrm{C}$ & 2.392225 & -2.0526 & 3.262277 \\
33 & $\mathrm{C}$ & 1.173422 & -2.05416 & 2.586296 \\
34 & $\mathrm{C}$ & -4.49897 & -1.7692 & 0.638264 \\
35 & $\mathrm{C}$ & 4.915753 & -1.06013 & -0.65895 \\
\hline
\end{tabular}

\begin{tabular}{|lllll|}
\hline 64 & $\mathrm{H}$ & 5.054001 & 0.845404 & 0.322574 \\
65 & $\mathrm{H}$ & 7.499328 & 0.666001 & 0.743508 \\
66 & $\mathrm{H}$ & 8.743385 & -1.35718 & 0.000071 \\
67 & $\mathrm{H}$ & -6.68967 & -4.24003 & 1.489995 \\
68 & $\mathrm{H}$ & -4.40205 & -3.46885 & 1.975367 \\
69 & $\mathrm{H}$ & 7.530243 & -3.18948 & -1.16651 \\
70 & $\mathrm{H}$ & 5.111703 & -3.00485 & -1.59021 \\
\hline
\end{tabular}


Formchk summary for $\mathbf{6 c}$.

Calculation Type $=$ FREQ. Calculation Method $=$ RB3LYP. Formula $\mathrm{C}_{39} \mathrm{H}_{25} \mathrm{~N}_{3} \mathrm{~S}_{3}$.

Basis Set $=6-31 G(D)$. Charge $=0$. Spin $=$ Singlet. Solvation $=$ None .

Total Energy $=-2860.0095$ a.u. Sum of Electronic and Thermal Free Energies $=-2859.5494$ a.u.

RMS Gradient Norm $=4 \cdot 27$ e-06 a.u. Imaginary Freq $=$ None .

Z-Matrix Cartesian coordinates.

\begin{tabular}{|c|c|c|c|c|}
\hline \multirow[t]{2}{*}{ Centre } & \multirow[t]{2}{*}{ Symbol } & \multicolumn{3}{|c|}{ Coordinates (Angstroms) } \\
\hline & & $x$ & Y & Z \\
\hline 1 & $\mathrm{~N}$ & -1.89047 & 2.20293 & 0.011405 \\
\hline 2 & C & -1.00117 & 1.144038 & -0.03058 \\
\hline 3 & C & 0.402588 & 1.533953 & 0.015884 \\
\hline 4 & s & 0.521462 & 3.288527 & -0.04975 \\
\hline 5 & C & -1.28866 & 3.347768 & -0.00135 \\
\hline 6 & C & -1.3376 & -0.18061 & -0.15703 \\
\hline 7 & C & -1.99357 & 4.633944 & 0.010844 \\
\hline 8 & C & 1.404551 & 0.62804 & 0.154369 \\
\hline 9 & $\mathrm{~N}$ & -2.61286 & -0.66196 & -0.12312 \\
\hline 10 & C & -2.66596 & -1.95597 & -0.02286 \\
\hline 11 & s & -1.0913 & -2.79847 & 0.10424 \\
\hline 12 & C & -0.22741 & -1.22498 & -0.43485 \\
\hline 13 & C & 1.049239 & -0.85386 & 0.419596 \\
\hline 14 & S & 2.637631 & -1.68336 & -0.13563 \\
\hline 15 & C & 3.494599 & -0.11762 & 0.02836 \\
\hline 16 & $\mathrm{~N}$ & 2.739945 & 0.93198 & 0.140232 \\
\hline 17 & C & 0.873536 & -1.11269 & 1.941566 \\
\hline 18 & C & 0.053847 & -1.3313 & -1.9605 \\
\hline 19 & C & -1.30631 & 5.858615 & 0.016951 \\
\hline 20 & C & -2.0088 & 7.061872 & 0.027296 \\
\hline 21 & C & -3.40468 & 7.058763 & 0.032908 \\
\hline 22 & C & -4.09699 & 5.843645 & 0.027808 \\
\hline 23 & C & -3.40116 & 4.639847 & 0.016509 \\
\hline 24 & C & 1.103201 & -2.39106 & 2.474786 \\
\hline 25 & C & 0.939766 & -2.64782 & 3.833149 \\
\hline 26 & C & 0.546967 & -1.62576 & 4.699717 \\
\hline 27 & C & 0.323121 & -0.35069 & 4.187261 \\
\hline 28 & c & 0.485614 & -0.09629 & 2.822882 \\
\hline
\end{tabular}

\begin{tabular}{|c|c|c|c|c|}
\hline \multirow[t]{2}{*}{ Centre } & \multirow[t]{2}{*}{ Symbol } & \multicolumn{3}{|c|}{ Coordinates (Angstroms) } \\
\hline & & $\mathrm{x}$ & Y & Z \\
\hline 36 & C & 5.585966 & 1.208701 & -0.03232 \\
\hline 37 & C & 6.972185 & 1.300185 & -0.07957 \\
\hline 38 & C & 7.755325 & 0.141466 & -0.10695 \\
\hline 39 & C & -3.96193 & -4.10315 & 0.044035 \\
\hline 40 & C & -5.17954 & -4.77709 & 0.104371 \\
\hline 41 & C & -6.37684 & -4.06034 & 0.148023 \\
\hline 42 & C & 7.140715 & -1.11169 & -0.08484 \\
\hline 43 & C & 5.751877 & -1.20976 & -0.03576 \\
\hline 44 & C & -6.35104 & -2.66182 & 0.13084 \\
\hline 45 & C & -5.13929 & -1.98335 & 0.072063 \\
\hline 46 & H & -0.21988 & 5.875939 & 0.016391 \\
\hline 47 & $\mathrm{H}$ & -1.46459 & 8.002081 & 0.032094 \\
\hline 48 & $\mathrm{H}$ & -3.9512 & 7.997902 & 0.041374 \\
\hline 49 & $\mathrm{H}$ & -5.18354 & 5.836477 & 0.031982 \\
\hline 50 & H & -3.92339 & 3.689358 & 0.011105 \\
\hline 51 & $\mathrm{H}$ & 1.427805 & -3.19633 & 1.823513 \\
\hline 52 & $\mathrm{H}$ & 1.125269 & -3.64844 & 4.214416 \\
\hline 53 & $\mathrm{H}$ & 0.424456 & -1.82215 & 5.761459 \\
\hline 54 & $\mathrm{H}$ & 0.025618 & 0.46026 & 4.846662 \\
\hline 55 & $\mathrm{H}$ & 0.316724 & 0.908755 & 2.456869 \\
\hline 56 & $\mathrm{H}$ & -0.63356 & 0.651903 & -2.46876 \\
\hline 57 & H & -0.15565 & 0.45131 & -4.86059 \\
\hline 58 & $\mathrm{H}$ & 0.797159 & -1.65574 & -5.78712 \\
\hline 59 & $\mathrm{H}$ & 1.257672 & -3.56328 & -4.24759 \\
\hline 60 & H & 0.768644 & -3.36651 & -1.85375 \\
\hline 61 & H & 4.966717 & 2.098716 & -0.01374 \\
\hline 62 & $\mathrm{H}$ & 7.446283 & 2.277747 & -0.09633 \\
\hline 63 & $\mathrm{H}$ & 8.838737 & 0.216277 & -0.14414 \\
\hline
\end{tabular}




\begin{tabular}{|lllll|}
\hline 29 & $C$ & -0.20597 & -0.27183 & -2.83798 \\
30 & $C$ & 0.061755 & -0.38744 & -4.20467 \\
31 & $C$ & 0.592729 & -1.56507 & -4.7237 \\
32 & $C$ & 0.850151 & -2.63283 & -3.86136 \\
33 & $C$ & 0.579648 & -2.51599 & -2.501 \\
34 & $C$ & -3.92614 & -2.69819 & 0.028969 \\
35 & $C$ & 4.956828 & -0.05126 & -0.01107 \\
\hline
\end{tabular}

\begin{tabular}{|lllll|}
\hline 64 & $\mathrm{H}$ & -3.03683 & -4.6704 & -0.00422 \\
65 & $\mathrm{H}$ & -5.19304 & -5.8634 & 0.113468 \\
66 & $\mathrm{H}$ & -7.32582 & -4.58771 & 0.194236 \\
67 & $\mathrm{H}$ & 7.74318 & -2.01559 & -0.10172 \\
68 & $\mathrm{H}$ & 5.284405 & -2.18967 & -0.00349 \\
69 & $\mathrm{H}$ & -7.28068 & -2.10027 & 0.164572 \\
70 & $\mathrm{H}$ & -5.10242 & -0.89958 & 0.061174 \\
\hline
\end{tabular}


Formchk summary for $\mathbf{7 0}$.

Calculation Type $=$ FREQ. Calculation Method $=$ RB3LYP. Formula $\mathrm{C}_{33} \mathrm{H}_{27} \mathrm{NS}_{3}$.

Basis Set $=6-31 G(D)$. Charge $=0$. Spin $=$ Singlet. Solvation $=$ None .

Total Energy $=-2523.118$ a.u. Sum of Electronic and Thermal Free Energies $=-2522.6816$ a.u.

RMS Gradient Norm = 7.099e-06 a.u. Imaginary Freq $=$ None.

Z-Matrix Cartesian coordinates.

\begin{tabular}{|c|c|c|c|c|}
\hline \multirow[t]{2}{*}{ Centre } & \multirow[t]{2}{*}{ Symbol } & \multicolumn{3}{|c|}{ Coordinates (Angstroms) } \\
\hline & & $x$ & $\mathrm{Y}$ & Z \\
\hline 1 & $\mathrm{~N}$ & -1.52944 & 2.016863 & -0.29519 \\
\hline 2 & C & -0.73223 & 0.896946 & -0.18004 \\
\hline 3 & C & 0.561841 & 1.132008 & 0.256496 \\
\hline 4 & $S$ & 0.767646 & 2.856434 & 0.511306 \\
\hline 5 & C & -0.90461 & 3.11864 & 0.020441 \\
\hline 6 & C & -1.32315 & -0.4142 & -0.53183 \\
\hline 7 & C & -1.51523 & 4.454148 & -0.03828 \\
\hline 8 & C & 1.649679 & 0.192573 & 0.574059 \\
\hline 9 & C & -2.56736 & -0.88215 & 0.043863 \\
\hline 10 & C & -2.93905 & -2.11779 & -0.43792 \\
\hline 11 & $S$ & -1.77114 & -2.70651 & -1.60855 \\
\hline 12 & C & -0.77881 & -1.28733 & -1.44761 \\
\hline 13 & C & 1.51003 & -0.82454 & 1.497079 \\
\hline 14 & $S$ & 2.975717 & -1.74698 & 1.621396 \\
\hline 15 & C & 3.797738 & -0.75328 & 0.431939 \\
\hline 16 & C & 2.965401 & 0.241149 & -0.03323 \\
\hline 17 & C & 0.326458 & -1.17565 & 2.347878 \\
\hline 18 & C & 0.456853 & -1.14359 & -2.28574 \\
\hline 19 & C & 5.193076 & -1.06403 & 0.073829 \\
\hline 20 & C & -4.11619 & -2.94531 & -0.11802 \\
\hline 21 & C & -3.98712 & -4.32882 & 0.10427 \\
\hline 22 & C & -5.10104 & -5.11496 & 0.392395 \\
\hline 23 & C & -6.36965 & -4.53694 & 0.471435 \\
\hline 24 & C & -6.51448 & -3.16647 & 0.249029 \\
\hline 25 & C & -5.40276 & -2.37996 & -0.05021 \\
\hline 26 & C & 5.604085 & -2.39312 & -0.13849 \\
\hline 27 & C & 6.925939 & -2.69029 & -0.46373 \\
\hline 28 & C & 7.867293 & -1.66679 & -0.59065 \\
\hline
\end{tabular}

\begin{tabular}{|c|c|c|c|c|}
\hline \multirow[t]{2}{*}{ Centre } & \multirow[t]{2}{*}{ Symbol } & \multicolumn{3}{|c|}{ Coordinates (Angstroms) } \\
\hline & & $\mathrm{x}$ & Y & Z \\
\hline 33 & C & -2.74443 & 6.976414 & -0.17676 \\
\hline 34 & C & -1.44596 & 6.85542 & 0.322053 \\
\hline 35 & C & -0.83568 & 5.60507 & 0.392247 \\
\hline 36 & H & -0.26437 & -0.27957 & 2.561007 \\
\hline 37 & H & -0.33514 & -1.89331 & 1.84767 \\
\hline 38 & $\mathrm{H}$ & 0.63758 & -1.61364 & 3.302043 \\
\hline 39 & $\mathrm{H}$ & 0.674135 & -0.08539 & -2.45863 \\
\hline 40 & $\mathrm{H}$ & 1.335944 & -1.58582 & -1.80131 \\
\hline 41 & $\mathrm{H}$ & 0.333731 & -1.62723 & -3.26069 \\
\hline 42 & H & -3.00102 & -4.78351 & 0.06456 \\
\hline 43 & H & -4.97584 & -6.18096 & 0.563597 \\
\hline 44 & $\mathrm{H}$ & -7.23753 & -5.14959 & 0.699859 \\
\hline 45 & $\mathrm{H}$ & -7.49887 & -2.70783 & 0.294578 \\
\hline 46 & $\mathrm{H}$ & -5.53039 & -1.32225 & -0.25819 \\
\hline 47 & $\mathrm{H}$ & 4.873913 & -3.19421 & -0.06274 \\
\hline 48 & $\mathrm{H}$ & 7.218988 & -3.72409 & -0.62664 \\
\hline 49 & $\mathrm{H}$ & 8.897129 & -1.89869 & -0.84807 \\
\hline 50 & $\mathrm{H}$ & 8.202707 & 0.45993 & -0.46237 \\
\hline 51 & $\mathrm{H}$ & 5.87048 & 0.983256 & 0.155288 \\
\hline 52 & $\mathrm{H}$ & -3.34231 & 3.694014 & -0.87424 \\
\hline 53 & $\mathrm{H}$ & -4.43901 & 5.922614 & -0.99715 \\
\hline 54 & $\mathrm{H}$ & -3.21957 & 7.952099 & -0.23054 \\
\hline 55 & $\mathrm{H}$ & -0.90654 & 7.735938 & 0.660043 \\
\hline 56 & $\mathrm{H}$ & 0.173337 & 5.526515 & 0.789069 \\
\hline 57 & C & -3.31847 & -0.1132 & 1.099689 \\
\hline 58 & C & 3.340705 & 1.231752 & -1.10576 \\
\hline 59 & $\mathrm{H}$ & -4.04253 & -0.7493 & 1.614682 \\
\hline 60 & $\mathrm{H}$ & -2.62972 & 0.295167 & 1.847822 \\
\hline
\end{tabular}




\begin{tabular}{|lllll|}
\hline 29 & $C$ & 7.475759 & -0.34377 & -0.37921 \\
30 & $C$ & 6.155937 & -0.04497 & -0.04344 \\
31 & $C$ & -2.8226 & 4.585175 & -0.53968 \\
32 & $C$ & -3.42875 & 5.836057 & -0.60619 \\
\hline
\end{tabular}

\begin{tabular}{|lllll|}
\hline 61 & $\mathrm{H}$ & -3.85115 & 0.743054 & 0.670776 \\
62 & $\mathrm{H}$ & 2.501142 & 1.406016 & -1.78692 \\
63 & $\mathrm{H}$ & 3.616542 & 2.209369 & -0.68965 \\
64 & $\mathrm{H}$ & 4.189529 & 0.873065 & -1.69363 \\
\hline
\end{tabular}


Formchk summary for $\mathbf{7 c}$.

Calculation Type $=$ FREQ. Calculation Method $=$ RB3LYP. Formula $\mathrm{C}_{33} \mathrm{H}_{27} \mathrm{NS}_{3}$.

Basis Set $=6-31 G(D)$. Charge $=0$. Spin $=$ Singlet. Solvation $=$ None .

Total Energy $=-2523.0865$ a.u. Sum of Electronic and Thermal Free Energies $=-2522.6441$ a.u.

RMS Gradient Norm $=1.2193 \mathrm{e}-05$ a.u. Imaginary Freq $=$ None.

Z-Matrix Cartesian coordinates.

\begin{tabular}{|c|c|c|c|c|}
\hline \multirow[t]{2}{*}{ Centre } & \multirow[t]{2}{*}{ Symbol } & \multicolumn{3}{|c|}{ Coordinates (Angstroms) } \\
\hline & & $x$ & Y & Z \\
\hline 1 & $\mathrm{~N}$ & 1.830553 & 2.00997 & 0.031037 \\
\hline 2 & C & 0.926797 & 0.960169 & 0.071447 \\
\hline 3 & C & -0.48557 & 1.375759 & 0.044286 \\
\hline 4 & s & -0.53663 & 3.148951 & 0.135127 \\
\hline 5 & C & 1.263135 & 3.169785 & 0.064285 \\
\hline 6 & C & 1.266341 & -0.36975 & 0.163062 \\
\hline 7 & C & 1.997152 & 4.440413 & 0.05709 \\
\hline 8 & C & -1.50894 & 0.484923 & -0.09957 \\
\hline 9 & C & 2.564943 & -0.99528 & 0.133869 \\
\hline 10 & C & 2.496608 & -2.36438 & 0.034469 \\
\hline 11 & s & 0.84295 & -3.0173 & -0.02292 \\
\hline 12 & C & 0.124173 & -1.35687 & 0.427715 \\
\hline 13 & C & -1.10089 & -0.96477 & -0.42565 \\
\hline 14 & $\mathrm{~S}$ & -2.62751 & -1.97102 & -0.05197 \\
\hline 15 & C & -3.64387 & -0.50694 & -0.06382 \\
\hline 16 & C & -2.94338 & 0.670569 & -0.07169 \\
\hline 17 & C & -0.85438 & -1.05976 & -1.9534 \\
\hline 18 & C & -0.15609 & -1.36975 & 1.953574 \\
\hline 19 & C & -5.10481 & -0.7035 & -0.04492 \\
\hline 20 & C & 3.603229 & -3.33394 & -0.05309 \\
\hline 21 & C & 4.696323 & -3.11338 & -0.91215 \\
\hline 22 & C & 5.733292 & -4.04064 & -0.99977 \\
\hline 23 & C & 5.699008 & -5.21003 & -0.23808 \\
\hline 24 & C & 4.613413 & -5.44929 & 0.607306 \\
\hline 25 & C & 3.573767 & -4.5264 & 0.69499 \\
\hline 26 & C & -5.94734 & 0.006369 & -0.92007 \\
\hline 27 & C & -7.32678 & -0.1944 & -0.90207 \\
\hline 28 & c & -7.89289 & -1.11377 & -0.01719 \\
\hline
\end{tabular}

\begin{tabular}{|c|c|c|c|c|}
\hline \multirow[t]{2}{*}{ Centre } & \multirow[t]{2}{*}{ Symbol } & \multicolumn{3}{|c|}{ Coordinates (Angstroms) } \\
\hline & & $x$ & Y & Z \\
\hline 33 & C & 3.458534 & 6.838814 & 0.044101 \\
\hline 34 & C & 4.124598 & 5.609692 & 0.01056 \\
\hline 35 & C & 3.403933 & 4.420469 & 0.017368 \\
\hline 36 & C & 3.848871 & -0.21147 & 0.238686 \\
\hline 37 & C & -3.57363 & 2.033607 & 0.058902 \\
\hline 38 & H & -1.745 & -0.7066 & -2.47979 \\
\hline 39 & $\mathrm{H}$ & -0.00655 & -0.43149 & -2.24389 \\
\hline 40 & H & -0.65053 & -2.08687 & -2.26211 \\
\hline 41 & $\mathrm{H}$ & -0.51581 & -0.39116 & 2.285982 \\
\hline 42 & $\mathrm{H}$ & -0.90258 & -2.12302 & 2.213339 \\
\hline 43 & $\mathrm{H}$ & 0.77241 & -1.59737 & 2.483782 \\
\hline 44 & $\mathrm{H}$ & 4.71524 & -2.22373 & -1.53331 \\
\hline 45 & $\mathrm{H}$ & 6.564706 & -3.85295 & -1.67404 \\
\hline 46 & H & 6.507363 & -5.93272 & -0.30861 \\
\hline 47 & H & 4.575253 & -6.35828 & 1.201693 \\
\hline 48 & $\mathrm{H}$ & 2.734627 & -4.71655 & 1.357429 \\
\hline 49 & $\mathrm{H}$ & -5.5117 & 0.697539 & -1.63504 \\
\hline 50 & $\mathrm{H}$ & -7.95872 & 0.360826 & -1.59017 \\
\hline 51 & $\mathrm{H}$ & -8.96791 & -1.27141 & -0.0059 \\
\hline 52 & H & -7.49784 & -2.55694 & 1.53698 \\
\hline 53 & $\mathrm{H}$ & -5.0538 & -2.19744 & 1.512255 \\
\hline 54 & $\mathrm{H}$ & 0.251606 & 5.719641 & 0.117343 \\
\hline 55 & $\mathrm{H}$ & 1.538425 & 7.819442 & 0.109231 \\
\hline 56 & $\mathrm{H}$ & 4.023988 & 7.766655 & 0.03917 \\
\hline 57 & H & 5.210409 & 5.579988 & -0.02036 \\
\hline 58 & H & 3.909552 & 3.461504 & -0.00762 \\
\hline 59 & H & 4.697124 & -0.86876 & 0.442227 \\
\hline 60 & $\mathrm{H}$ & 4.055336 & 0.347228 & -0.68212 \\
\hline
\end{tabular}




\begin{tabular}{|lllll|}
\hline 29 & $\mathrm{C}$ & -7.06718 & -1.8366 & 0.84663 \\
30 & $\mathrm{C}$ & -5.68847 & -1.63795 & 0.830726 \\
31 & $\mathrm{C}$ & 1.336998 & 5.679818 & 0.089246 \\
32 & $\mathrm{C}$ & 2.063685 & 6.868767 & 0.083662 \\
\hline
\end{tabular}

\begin{tabular}{|lllll|}
\hline 61 & $\mathrm{H}$ & 3.777096 & 0.532147 & 1.037156 \\
62 & $\mathrm{H}$ & -3.1448 & 2.575366 & 0.910594 \\
63 & $\mathrm{H}$ & -3.40596 & 2.65122 & -0.83238 \\
64 & $\mathrm{H}$ & -4.65019 & 1.962128 & 0.223966 \\
\hline
\end{tabular}


Formchk summary for $\mathbf{8 0}$.

Calculation Type $=$ FREQ. Calculation Method $=$ RB3LYP. Formula $\mathrm{C}_{43} \mathrm{H}_{31} \mathrm{NS}_{3}$.

Basis Set $=6-31 G(D)$. Charge $=0$. Spin $=$ Singlet. Solvation $=$ None .

Total Energy $=-2906.5928$ a.u. Sum of Electronic and Thermal Free Energies $=-2672.7237$ a.u.

RMS Gradient Norm $=2.454 \mathrm{e}-06$ a.u. Imaginary Freq $=$ None .

Z-Matrix Cartesian coordinates.

\begin{tabular}{|c|c|c|c|c|}
\hline \multirow[t]{2}{*}{ Centre } & \multirow[t]{2}{*}{ Symbol } & \multicolumn{3}{|c|}{ Coordinates (Angstroms) } \\
\hline & & $x$ & $\mathrm{Y}$ & Z \\
\hline 1 & C & 0.885183 & 1.108343 & 0.23106 \\
\hline 2 & C & -0.20499 & 1.372152 & -0.57637 \\
\hline 3 & C & 1.365682 & 3.252707 & -0.18754 \\
\hline 4 & $S$ & -0.13304 & 3.047409 & -1.09188 \\
\hline 5 & C & 1.203868 & -0.19833 & 0.864198 \\
\hline 6 & C & 0.477156 & -0.75877 & 1.89533 \\
\hline 7 & C & 2.398085 & -2.20242 & 1.124247 \\
\hline 8 & $\mathrm{~S}$ & 1.124562 & -2.32358 & 2.320346 \\
\hline 9 & C & -1.30615 & 0.484482 & -1.00394 \\
\hline 10 & C & -1.13811 & -0.56892 & -1.88602 \\
\hline 11 & C & -3.48626 & -0.3917 & -0.97848 \\
\hline 12 & $S$ & -2.62736 & -1.45661 & -2.06947 \\
\hline 13 & C & 3.358084 & -3.31159 & 0.978739 \\
\hline 14 & C & 3.909573 & -3.94219 & 2.109301 \\
\hline 15 & C & 3.730859 & -3.78239 & -0.29366 \\
\hline 16 & C & 4.806619 & -4.99971 & 1.97246 \\
\hline 17 & $\mathrm{H}$ & 3.642837 & -3.58536 & 3.100321 \\
\hline 18 & C & 4.636866 & -4.8338 & -0.42751 \\
\hline 19 & $\mathrm{H}$ & 3.286615 & -3.33477 & -1.17725 \\
\hline 20 & C & 5.178485 & -5.44761 & 0.703281 \\
\hline 21 & $\mathrm{H}$ & 5.222803 & -5.46893 & 2.860109 \\
\hline 22 & $\mathrm{H}$ & 4.910728 & -5.18246 & -1.42005 \\
\hline 23 & $\mathrm{H}$ & 5.881301 & -6.26955 & 0.59682 \\
\hline 24 & C & -4.9091 & -0.65547 & -0.69069 \\
\hline 25 & c & -5.81157 & -0.94668 & -1.72959 \\
\hline 26 & C & -5.39205 & -0.64863 & 0.630511 \\
\hline 27 & C & -7.15238 & -1.21267 & -1.45783 \\
\hline 28 & $\mathrm{H}$ & -5.45786 & -0.94744 & -2.757 \\
\hline
\end{tabular}

\begin{tabular}{|c|c|c|c|c|}
\hline \multirow[t]{2}{*}{ Centre } & \multirow[t]{2}{*}{ Symbol } & \multicolumn{3}{|c|}{ Coordinates (Angstroms) } \\
\hline & & $\mathrm{x}$ & Y & Z \\
\hline 40 & C & 1.813993 & -0.41038 & -4.25028 \\
\hline 41 & $\mathrm{H}$ & 0.478696 & 1.027705 & -3.36936 \\
\hline 42 & C & 2.158905 & -1.75922 & -4.36218 \\
\hline 43 & H & 1.696607 & -3.76813 & -3.72268 \\
\hline 44 & $\mathrm{H}$ & 2.36213 & 0.341082 & -4.81234 \\
\hline 45 & $\mathrm{H}$ & 2.978705 & -2.06265 & -5.0076 \\
\hline 46 & C & -0.61804 & -0.19624 & 2.705556 \\
\hline 47 & C & -1.71934 & -0.98324 & 3.085998 \\
\hline 48 & C & -0.54349 & 1.12624 & 3.180443 \\
\hline 49 & C & -2.71421 & -0.46496 & 3.914883 \\
\hline 50 & $\mathrm{H}$ & -1.7962 & -2.00368 & 2.721048 \\
\hline 51 & C & -1.54295 & 1.643138 & 4.004061 \\
\hline 52 & $\mathrm{H}$ & 0.314882 & 1.736653 & 2.917919 \\
\hline 53 & C & -2.63146 & 0.850746 & 4.376501 \\
\hline 54 & H & -3.5541 & -1.0925 & 4.201985 \\
\hline 55 & $\mathrm{H}$ & -1.46341 & 2.664822 & 4.366163 \\
\hline 56 & $\mathrm{H}$ & -3.40503 & 1.253154 & 5.024923 \\
\hline 57 & C & 2.096386 & 4.526389 & -0.13419 \\
\hline 58 & C & 1.648009 & 5.673243 & -0.80918 \\
\hline 59 & C & 3.283642 & 4.600587 & 0.616238 \\
\hline 60 & C & 2.367461 & 6.863962 & -0.73671 \\
\hline 61 & $\mathrm{H}$ & 0.732738 & 5.63901 & -1.39477 \\
\hline 62 & C & 3.998947 & 5.792243 & 0.685244 \\
\hline 63 & $\mathrm{H}$ & 3.625605 & 3.712918 & 1.13718 \\
\hline 64 & C & 3.545442 & 6.928764 & 0.010064 \\
\hline 65 & H & 2.006643 & 7.742235 & -1.26489 \\
\hline 66 & H & 4.914624 & 5.835012 & 1.268869 \\
\hline 67 & $\mathrm{H}$ & 4.105807 & 7.85801 & 0.065668 \\
\hline
\end{tabular}




\begin{tabular}{|lllll|}
\hline 29 & $\mathrm{C}$ & -6.73621 & -0.90526 & 0.898261 \\
30 & $\mathrm{H}$ & -4.70179 & -0.46429 & 1.448163 \\
31 & $\mathrm{C}$ & -7.6218 & -1.18845 & -0.14312 \\
32 & $\mathrm{H}$ & -7.83316 & -1.4314 & -2.27628 \\
33 & $\mathrm{H}$ & -7.08948 & -0.89613 & 1.926181 \\
34 & $\mathrm{H}$ & -8.66803 & -1.39231 & 0.068197 \\
35 & $\mathrm{C}$ & 0.026302 & -0.97178 & -2.69692 \\
36 & $\mathrm{C}$ & 0.384545 & -2.3263 & -2.81653 \\
37 & $\mathrm{C}$ & 0.75717 & -0.01924 & -3.42927 \\
38 & $\mathrm{C}$ & 1.439003 & -2.71529 & -3.64218 \\
39 & $\mathrm{H}$ & -0.1616 & -3.07395 & -2.24846 \\
\hline
\end{tabular}

\begin{tabular}{|lllll|}
\hline 68 & $\mathrm{~N}$ & 1.74857 & 2.165405 & 0.427751 \\
69 & $\mathrm{C}$ & 3.313068 & -0.542 & -0.59377 \\
70 & $\mathrm{H}$ & 2.913173 & -0.6212 & -1.61082 \\
71 & $\mathrm{H}$ & 3.565226 & 0.509515 & -0.42389 \\
72 & $\mathrm{H}$ & 4.233779 & -1.12891 & -0.54764 \\
73 & $\mathrm{C}$ & -3.10733 & 1.719657 & 0.402294 \\
74 & $\mathrm{H}$ & -4.19576 & 1.738626 & 0.490588 \\
75 & $\mathrm{H}$ & -2.68574 & 1.622843 & 1.408213 \\
76 & $\mathrm{H}$ & -2.78651 & 2.691967 & 0.012846 \\
77 & $\mathrm{C}$ & 2.320176 & -1.00821 & 0.43882 \\
78 & $\mathrm{C}$ & -2.65821 & 0.601575 & -0.50161 \\
\hline
\end{tabular}


Formchk summary for $\mathbf{8 c}$.

Calculation Type $=$ FREQ. Calculation Method $=$ RB3LYP. Formula $\mathrm{C}_{43} \mathrm{H}_{31} \mathrm{NS}_{3}$.

Basis Set $=6-31 G(D)$. Charge $=0$. Spin $=$ Singlet. Solvation $=$ None .

Total Energy $=-2906.5342$ a.u. Sum of Electronic and Thermal Free Energies $=-2672.6718$ a.u.

RMS Gradient Norm $=4.382 \mathrm{e}-06$ a.u. Imaginary Freq $=$ None.

Z-Matrix Cartesian coordinates.

\begin{tabular}{|c|c|c|c|c|}
\hline \multirow[t]{2}{*}{ Centre } & \multirow[t]{2}{*}{ Symbol } & \multicolumn{3}{|c|}{ Coordinates (Angstroms) } \\
\hline & & $x$ & Y & Z \\
\hline 1 & C & -1.08718 & 1.162909 & -0.03587 \\
\hline 2 & C & 0.300529 & 1.643611 & -0.00682 \\
\hline 3 & C & -1.52755 & 3.354037 & -0.00797 \\
\hline 4 & s & 0.270846 & 3.417188 & -0.09897 \\
\hline 5 & C & -1.36385 & -0.17743 & -0.16334 \\
\hline 6 & C & -0.18711 & -1.1189 & -0.45933 \\
\hline 7 & C & -2.53428 & -2.2057 & -0.06011 \\
\hline 8 & $\mathrm{~S}$ & -0.86903 & -2.80083 & 0.012301 \\
\hline 9 & C & 1.364278 & 0.805337 & 0.136777 \\
\hline 10 & C & 1.052206 & -0.67978 & 0.413895 \\
\hline 11 & C & 3.56338 & -0.02243 & 0.059633 \\
\hline 12 & s & 2.646675 & -1.53504 & -0.08016 \\
\hline 13 & C & -3.60795 & -3.21366 & 0.01125 \\
\hline 14 & C & -3.55154 & -4.3772 & -0.77877 \\
\hline 15 & C & -4.69338 & -3.05594 & 0.892771 \\
\hline 16 & C & -4.56034 & -5.33536 & -0.70784 \\
\hline 17 & $\mathrm{H}$ & -2.71817 & -4.51479 & -1.46114 \\
\hline 18 & C & -5.69909 & -4.01853 & 0.963395 \\
\hline 19 & $\mathrm{H}$ & -4.73016 & -2.1874 & 1.542532 \\
\hline 20 & C & -5.63913 & -5.15976 & 0.161527 \\
\hline 21 & $\mathrm{H}$ & -4.50384 & -6.22138 & -1.33458 \\
\hline 22 & $\mathrm{H}$ & -6.52598 & -3.88056 & 1.655011 \\
\hline 23 & $\mathrm{H}$ & -6.42324 & -5.90989 & 0.218644 \\
\hline 24 & C & 5.03428 & -0.12195 & 0.023928 \\
\hline 25 & C & 5.707264 & -1.0768 & 0.808732 \\
\hline 26 & C & 5.796875 & 0.709479 & -0.81689 \\
\hline 27 & C & 7.096432 & -1.17645 & 0.772783 \\
\hline 28 & $\mathrm{H}$ & 5.131779 & -1.72893 & 1.458793 \\
\hline
\end{tabular}

\begin{tabular}{|c|c|c|c|c|}
\hline \multirow[t]{2}{*}{ Centre } & \multirow[t]{2}{*}{ Symbol } & \multicolumn{3}{|c|}{ Coordinates (Angstroms) } \\
\hline & & $x$ & Y & Z \\
\hline 40 & C & -4.49909 & 5.65731 & 0.072302 \\
\hline 41 & H & -4.18516 & 3.521408 & 0.060735 \\
\hline 42 & C & -3.89052 & 6.916297 & 0.057911 \\
\hline 43 & $\mathrm{H}$ & -2.01774 & 7.98564 & 0.013195 \\
\hline 44 & $\mathrm{H}$ & -5.58242 & 5.576957 & 0.099403 \\
\hline 45 & H & -4.49837 & 7.816797 & 0.074029 \\
\hline 46 & $\mathrm{~N}$ & -2.03962 & 2.168439 & 0.023782 \\
\hline 47 & C & -3.95345 & -0.10045 & -0.24259 \\
\hline 48 & $\mathrm{H}$ & -4.7795 & -0.78923 & -0.43292 \\
\hline 49 & $\mathrm{H}$ & -4.16896 & 0.457122 & 0.676403 \\
\hline 50 & $\mathrm{H}$ & -3.91769 & 0.638391 & -1.04821 \\
\hline 51 & C & 3.32084 & 2.494944 & 0.323063 \\
\hline 52 & $\mathrm{H}$ & 4.391107 & 2.482009 & 0.540201 \\
\hline 53 & $\mathrm{H}$ & 3.171957 & 3.102684 & -0.57924 \\
\hline 54 & H & 2.817106 & 3.01168 & 1.147594 \\
\hline 55 & C & 2.784313 & 1.096138 & 0.159461 \\
\hline 56 & C & -2.64421 & -0.84099 & -0.14604 \\
\hline 57 & C & 0.104921 & -1.15065 & -1.99402 \\
\hline 58 & C & 0.730813 & -2.26613 & -2.57542 \\
\hline 59 & C & -0.25452 & -0.09653 & -2.84328 \\
\hline 60 & C & 1.002461 & -2.31868 & -3.93981 \\
\hline 61 & $\mathrm{H}$ & 0.999577 & -3.11625 & -1.95823 \\
\hline 62 & C & 0.012721 & -0.14699 & -4.21418 \\
\hline 63 & $\mathrm{H}$ & -0.75901 & 0.775736 & -2.44804 \\
\hline 64 & C & 0.644459 & -1.2554 & -4.77083 \\
\hline 65 & $\mathrm{H}$ & 1.490806 & -3.19723 & -4.3531 \\
\hline 66 & $\mathrm{H}$ & -0.28521 & 0.687659 & -4.84354 \\
\hline 67 & $\mathrm{H}$ & 0.849181 & -1.29675 & -5.83739 \\
\hline
\end{tabular}




\begin{tabular}{|lllll|}
\hline 29 & $\mathrm{C}$ & 7.186599 & 0.605506 & -0.85196 \\
30 & $\mathrm{H}$ & 5.291809 & 1.419614 & -1.46417 \\
31 & $\mathrm{C}$ & 7.842353 & -0.33483 & -0.05532 \\
32 & $\mathrm{H}$ & 7.597776 & -1.91334 & 1.394652 \\
33 & $\mathrm{H}$ & 7.756679 & 1.253621 & -1.51234 \\
34 & $\mathrm{H}$ & 8.925518 & -0.41637 & -0.08504 \\
35 & $\mathrm{C}$ & -2.31968 & 4.588613 & 0.015725 \\
36 & $\mathrm{C}$ & -1.71744 & 5.857605 & 0.003396 \\
37 & $\mathrm{C}$ & -3.7242 & 4.502896 & 0.050982 \\
38 & $\mathrm{C}$ & -2.49845 & 7.011408 & 0.023253 \\
39 & $\mathrm{H}$ & -0.63493 & 5.94794 & -0.01854 \\
\hline
\end{tabular}

\begin{tabular}{|lllll|}
\hline 68 & $\mathrm{C}$ & 0.847383 & -0.90766 & 1.944592 \\
69 & $\mathrm{C}$ & 1.087904 & -2.1682 & 2.516121 \\
70 & $\mathrm{C}$ & 0.434707 & 0.121642 & 2.80041 \\
71 & $\mathrm{C}$ & 0.909106 & -2.39498 & 3.878195 \\
72 & $\mathrm{H}$ & 1.435499 & -2.98451 & 1.892793 \\
73 & $\mathrm{C}$ & 0.257469 & -0.10082 & 4.168688 \\
74 & $\mathrm{H}$ & 0.255233 & 1.115382 & 2.410464 \\
75 & $\mathrm{C}$ & 0.491052 & -1.35934 & 4.71595 \\
76 & $\mathrm{H}$ & 1.102825 & -3.38426 & 4.284378 \\
77 & $\mathrm{H}$ & -0.05878 & 0.722789 & 4.803646 \\
78 & $\mathrm{H}$ & 0.357227 & -1.53205 & 5.78059 \\
\hline
\end{tabular}




\section{References}

(1) Frisch, M. J.; Trucks, G.W.; Schlegel, H. B.; Scuseria, G. E.; Robb, M. A.; Cheeseman, J. R.; Scalmani, G.; Barone, V.; Mennucci, B.; Petersson, G. A.; Nakatsuji, H.; Caricato, M.; Li, X.; Hratchian, H. P.; Izmaylov, A. F.; Bloino, J.; Zheng, G.; Sonnenberg, J. L.; Hada, M.; Ehara, M.; Toyota, K.; Fukuda, R.; Hasegawa, J.; Ishida, M.; Nakajima, T.; Honda, Y.; Kitao, O.; Nakai, H.; Vreven, T.; Montgomery Jr, J. A.; Peralta, J. E.; Ogliaro, F.; Bearpark, M.; Heyd, J. J.; Brothers, E.; Kudin, K. N.; Staroverov, V. N.; Kobayashi, R.; Normand, J.; Raghavachari, K.; Rendell, A.; Burant, J. C.; Iyengar, S. S.; Tomasi, J.; Cossi, M.; Rega, N.; Millam, J. M.; Klene, M.; Knox, J. E.; Cross, J. B.; Bakken, V.; Adamo, C.; Jaramillo, J.; Gomperts, R.; Stratmann, R. E.; Yazyev, O.; Austin, A. J.; Cammi, R.; Pomelli, C.; Ochterski, J. W.; Martin, R. L.; Morokuma, K.; Zakrzewski, V. G.; Voth, G. A.; Salvador, P.; Dannenberg, J. J.; Dapprich, S.; Daniels, A. D.; Farkas, Ö.; Foresman, J. B.; Ortiz, J. V.; Cioslowski, J.; Fox, D. J. Gaussian 09 Revision A.02, Gaussian Inc. Wallingford CT, 2009.

(2) Kutsunugi, Y.; Kawai, S.; Nakashima, T.; Kawai, T. Photochromic properties of terarylene derivatives having a $\pi$-conjugation unit on central aromatic ring. New J. Chem. 2009, 33, 1368-1373.

(3) Fukumoto, S.; Nakashima, T.; Kawai, T. Photon-quantitative reaction of a dithiazolylarylene in solution. Angew. Chem. Int. Ed. 2011, 50, 1565-1568.

(4) Nakashima, T. et al. Efficient oxidative cycloreversion reaction of photochromic dithiazolythiazole. J. Am. Chem. Soc. 2012, 134, 19877-19883.

(5) Calupitan, J. P. D. C.; Nakashima, T.; Hashimoto, Y.; Kawai, T. Fast and efficient oxidative cycloreversion reaction of a $\pi$-extended photochromic terarylene. Chem. Eur. J. 2016, 22, 10002-10008.

(6) Asato, R. et al. Photosynergetic amplification of radiation input: from efficient UV induced cycloreversion to sensitive X-ray detection. Chem. Sci. 2020, 11, 2504-2510.

(7) Nakashima, T. et al. Photochromism of thiazole-containing triangle terarylenes. Eur. J. Org. Chem. 2007, 3212-3218.

(8) Kawai, S. et al. Photochromic amorphous molecular materials based on dibenzothienylthiazole structure. J. Mater. Chem. 2009, 19, 3606-3611.

(9) Li, R.; Nakashima, T.; Kawai, T. A self-contained photoacid generator for super acid based on photochromic terarylene. Chem. Commun. 2017, 53, 4339-4341.

(10) Kucharski, T. J.; Tian, Y.; Akbulatov, S.; Boulatov, R. Chemical solutions for the closed-cycle storage of solar energy. Energy Environ. Sci. 2011, 4, 4449-4472.

(11) Jorner, K. et al. J. Mater. Chem. A. 2017, 5, 12369-12378. 